\title{
Comments on orientifolds without vector structure
}

\author{
Constantin Bachas, ${ }^{a}$ Massimo Bianchi, ${ }^{b c}$ Ralph Blumenhagen, ${ }^{d}$ Dieter Lüst ${ }^{d e}$ and \\ Timo Weigand ${ }^{f}$ \\ ${ }^{a}$ Laboratoire de Physique Théorique de l'École Normale Supérieure, \\ 24 rue Lhomond, 75231 Paris, France* \\ ${ }^{b}$ Physics Department, Theory Unit, CERN, \\ CH1211, Geneva 23, Switzerland \\ "Dipartimento di Fisica $\&$ Sezione INFN, Università di Roma "Tor Vergata", \\ Via della Ricerca Scientifica, 00133 Roma, Italy \\ ${ }^{d}$ Max-Planck-Institut für Physik, \\ Föhringer Ring 6, 80805 München, Germany \\ e Arnold-Sommerfeld-Center for Theoretical Physics, \\ Department for Physics, Ludwig-Maximilians-Universität München, \\ Theresienstr. 37, 80333 München, Germany \\ ${ }^{f}$ Department of Physics and Astronomy, University of Pennsylvania, \\ 209 South 33rd Street, Philadelphia, PA 19104-6396, U.S.A. \\ E-mail: bachas@lpt.ens.fr, Massimo.Bianchi@roma2.infn.it, \\ blumenha@mppmu.mpg.de, dieter.luest@lmu.de, timo@sas.upenn.edu
}

Abstract: We revisit type I compactifications with a $\operatorname{Spin}(32) / \mathbb{Z}_{2}$ gauge bundle that admits no vector structure. We elucidate the relation of this $\mathbb{Z}_{2}$ obstruction to discrete $B$ field flux and to 't Hooft flux and clarify some subtleties in the T-duality transformation to type IIA intersecting D-brane models. We reexamine the earliest 3 -generation GUT model on magnetized D-branes and show its consistency when a discrete $B$-flux is switched on. We further generalize partially known results for toroidal models to type I compactifications without vector structure and their mirror dual type IIA orientifolds on genuine Calabi-Yau manifolds. We illustrate this by working out the example of the quintic in some detail.

KEYWORDS: Intersecting branes models, D-branes.

\footnotetext{
${ }^{*}$ Unité mixte de recherche (UMR 8549) du CNRS et de l'ENS, associée à l'Université Pierre et Marie Curie et aux fédérations de recherche FR684 et FR2687.
} 


\section{Contents}

1. Introduction 1

2. Gauge bundles and orientifolds on $T^{2}$

2.1 Long strings and 't Hooft flux 3

2.2 Relation to the $B$-flux

2.3 Including the orientifold

3. Toroidal 4d orientifolds and the model $\mathrm{C} \quad \mathbf{1 0}$

3.1 Consistency conditions for $T^{6} / \Omega \mathcal{R}$ orientifolds 10

3.2 Three generations and the model $\mathrm{C}$

3.3 Euclidean D1-brane instantons 13

4. Calabi-Yau compactifications of $\operatorname{Spin}(32) / \mathbb{Z}_{2}$ bundles with or without vector structure

$4.1 \operatorname{Spin}(32) / \mathbb{Z}_{2}$ gauge bundles

4.2 Smooth Calabi-Yau type IIA orientifolds 17

4.3 Example: the quintic 20

5. Outlook 21

A. Toroidal example 23

\section{Introduction}

The purpose of the present note is to clarify an issue arising in the study of compactifications of type I and heterotic string theories. Such compactifications are specified by the choice of an internal manifold, $X$, and of a gauge bundle over $X$ suitably embedded in $\operatorname{Spin}(32) / \mathbb{Z}_{2}$. Because the gauge group is $\operatorname{Spin}(32) / \mathbb{Z}_{2}$, rather than $\mathrm{SO}(32)$, certain choices which would be forbidden in the latter case are in fact allowed. These are the gauge bundles "without vector structure". They play an important role in the discussion of various string dualities, as pointed out some time ago in [1- 3] and further analyzed in [4-14].

A crucial ingredient of the discussion on the type I side is the option of turning on a non-zero but quantized background of the internal NS-NS 2-form $B_{i j}$, which is odd under the worldsheet parity $\Omega$. This was recognized early on, based on intuition gained from rational models [15-17], in the first systematic study of toroidal compactifications of the type I theory [18]. The key observation is that since the flux of $B$ through any 2-cycle $\gamma$ of $X$ is defined (in appropriate units) up to $2 \pi$ shifts, both $\int_{\gamma} B=0$ and $\int_{\gamma} B=\pi$ can be 
compatible with the $\Omega$ projection. These discrete closed-string moduli are thus described by an element of a $\bmod 2$ cohomology, $b \in H^{2}\left(X, \mathbb{Z}_{2}\right)$. A worldsheet argument [5] then shows that the gauge bundle, $E$, supported on the D9-branes of the type I theory must obey the consistency condition

$$
b=\tilde{w}_{2}(E),
$$

where $\tilde{w}_{2}$ is a generalized Stiefel-Whitney class which measures the obstruction to endowing $E$ with vector structure [1]. In the special case of toroidal models and flat $E$, a non-zero torsion class $b$ leads to unbroken gauge groups with reduced rank [18]. This statement acquires a more intuitive, geometric meaning when translated in the T-dual language of type IIA orientifolds, discussed in [8-10]. Here we will clarify the precise meaning of the consistency condition (1.1), and further elucidate the T-duality transformation and the reduction of the rank. For simplicity we will perform this analysis for the simplest case of compactifications on a single two-torus in section 2. This admits a straightforward generalization to four-dimensional models on $T^{2} \times T^{2} \times T^{2}$ as will be summarized in section 3 .

In a different development, one of us (CB) noted that type I theory on magnetized tori presented many interesting phenomenological features, which were illustrated with a simple (non-supersymmetric but only marginally unstable) grand-unified 3-generation model [19]. The systematic analysis of the model-building possibilities of type I magnetic fields, and of their T-dual intersecting D-branes, started with the work in [20-22] and has been very actively pursued thereafter (for reviews and more references see [23-27]). The 3-generation model of reference [19] (hereafter called for short "model C") was actually discarded in [20], as being T-dual to a type IIA orientifold with half-integer D6-brane wrapping numbers. Closer inspection, however, reveals that the magnetic fields of model $\mathrm{C}$ describe precisely a $\operatorname{Spin}(32) / \mathbb{Z}_{2}$ bundle without vector structure. As we will explain in section 3 , turning on the discrete $B$-field background required by condition (1.1) makes model $\mathrm{C}$ consistent, and restores the integrality of the D6-brane wrapping numbers in the IIA picture. Furthermore, contrary to the case of flat bundles, the rank of the unbroken gauge group is not reduced.

Part of our motivation in writing this note was the wish to clarify this subtle point, and to amend/rectify the relevant statements in [19, 20]. While the phenomenological advantages of discrete B-fields in type IIB orientifolds and their type IIA duals of tilted complex structures have been heavily used in toroidal model building beginning with [20] (for more references see [23-27]), our emphasis here is on working out the conceptual connection with absence of vector structure. Moreover, gauge bundles without vector structure have not been much used in model building on non-toroidal backgrounds so far. The simplicity of the gauge bundle of model $\mathrm{C}$, which yields quite readily a 3-generation grand-unified model, is an encouragement to further explore this direction.

As one step towards this aim we generalize this framework to type I compactifications without vector structure on genuine Calabi-Yau manifolds in section 4 . Their mirror dual type IIA orientifolds are distinguished by allowing for a non-vanishing real part of some of the complex structure moduli. While this freedom is usually not much appreciated in the literature it does amplify considerably the possibilities for model building. We conclude by illustrating these observations for the example of the quintic. 


\section{Gauge bundles and orientifolds on $T^{2}$}

We will first discuss gauge bundles without vector structure in the simplest case of a toroidal Type I compactification [18]. Although for such backgrounds most of the work on model-building has been already done, our discussion will hopefully shed some more light on a few subtle points. ${ }^{1}$ It also serves as preparation for the discussion of orientfiolds without vector structure on general Calabi-Yau spaces in section 4.

\subsection{Long strings and 't Hooft flux}

Consider compactification of type II string theory on a 2-torus parameterized by $\left(x^{8}, x^{9}\right) \in$ $[0,1] \times[0,1]$. The torus is wrapped by a stack of $n \mathrm{D}(2+k)$-branes which carry on their world-volume a $\mathrm{U}(n)$ gauge field $\left(A_{8}, A_{9}\right)$. In this section the extra $k$ dimensions of the D-branes will be inert, so we may as well set $k=0$. We are interested in configurations

$$
A_{8}=0 ; \quad A_{9}=\operatorname{diag}\left[f^{1}, \ldots, f^{n}\right] x^{8}+\operatorname{diag}\left[\alpha^{1}, \ldots, \alpha^{n}\right],
$$

corresponding to a constant diagonal magnetic field $F_{89}=\operatorname{diag}\left[f^{1}, \ldots f^{n}\right]$. This background field defines the field strength of a gauge bundle, and the observable gauge symmetry on the D-branes is the commutant of the structure group of this bundle in $\mathrm{U}(n)$ (modulo the issue of massive U(1) factors.) If there are no other D-branes in the problem, the first Chern class, which counts the number of D-particles, must be integer:

$$
m \equiv \frac{1}{2 \pi} \int_{T^{2}} \operatorname{tr} F_{89}=\sum_{I=1}^{n} \frac{f^{I}}{2 \pi} \in \mathbb{Z} .
$$

We would like to understand the quantization conditions for the individual $f^{I}$. The argument is well-known, but we summarize it here for completeness.

If the structure group of the gauge bundle were $\mathrm{U}(1)^{n}$, then standard Dirac quantization condition would impose that

$$
\frac{f^{I}}{2 \pi}=m^{I} \in \mathbb{Z} \quad \forall I
$$

But if we choose the structure group to fill the full $\mathrm{U}(n)$, these conditions are in fact too restrictive. One example of an allowed gauge bundle that violates them is

$$
f^{I}=\frac{2 \pi}{n} \quad \text { and } \quad \alpha^{I}=\frac{2 \pi I}{n} .
$$

As one can easily verify, this is a consistent configuration because

$$
A_{\mu}\left(x^{8}+1\right)=\mathcal{U}^{-1}\left[A_{\mu}\left(x^{8}\right)+i \partial_{\mu}\right] \mathcal{U}, \quad \text { with } \quad \mathcal{U}=e^{-2 \pi i \times \operatorname{diag}[1 \cdots 0] x^{9}} \mathcal{P}
$$

Here $\mathcal{P}$ is the cyclic-shift permutation that sends $I \rightarrow I+1$, and the gauge transformation $\mathcal{U}$ is periodic when $x^{9} \rightarrow x^{9}+1$, as it should be. Notice that the transition functions $\mathcal{U}$ cannot

\footnotetext{
${ }^{1}$ See also 28 for recent work on the subject from a different vantage point.
} 

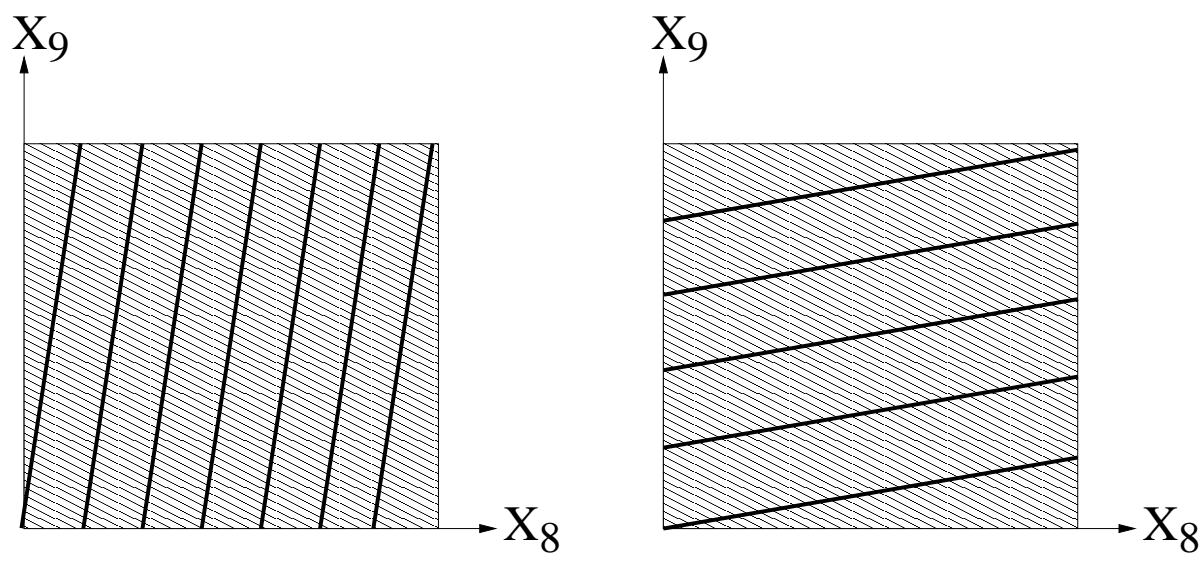

Figure 1: The left figure shows the T-dual of an abelian bundle $(n, m)=(1,7)$ and the right image the T-dual of a 't Hooft bundle $(n, m)=(5,1)$.

be chosen in $\mathrm{U}(1)^{n}$, except when the fluxes $m^{I}$ are integer. Other consistent non-abelian gauge bundles, with fractional fluxes $f^{I} \in 2 \pi \mathbb{Z} / n$, can be constructed in a similar way.

These gauge-theory statements acquire a simple geometric meaning after a T-duality in the $x^{9}$ direction. The duality transforms the D2-branes to D-strings and sends $A_{9} \rightarrow 2 \pi Y^{9}$, where $Y^{9}$ is the position of the D-strings in the transverse dualized dimension. As shown in figure 1, the abelian bundles (2.3) get mapped to configurations of $N$ independent D-strings with integer winding numbers $\left(1, m^{I}\right)$. Furthermore, their transverse positions $\alpha^{I} / 2 \pi$ are unconstrained, and the unbroken gauge symmetry has rank $n$. The configuration T-dual to (2.4), on the other hand, has $n$ pieces of D-string with fractional winding number $1 / n$ on the dual torus. These combine suitably so as to form a single "long D-string" winding $(n, 1)$ times in the $\left(x^{8}, \tilde{x}^{9}\right)$ directions. Such long-string configurations are familiar from the counting of black-hole microstates and from the Matrix-model proposal for M theory, see for example [29, 30]. Notice that the above long D-string is the minimal-energy configuration in the $(n, 1)$ topological sector.

It is instructive for our purposes here to separate the transition function (2.5) into a $\mathrm{U}(1)$ phase and an $\mathrm{SU}(n)$ part, i.e. to write

$$
\mathcal{U}=e^{-2 \pi i x^{9} / n} e^{-i \pi / n} \widehat{\mathcal{U}} \quad \text { with } \quad \widehat{\mathcal{U}}\left(x^{9}\right) \in \mathrm{SU}(n) .
$$

Neither of the two factors is periodic when $x^{9} \rightarrow x^{9}+1$, but the phases $e^{\mp 2 \pi i / n}$ that they acquire cancel in the product. As a result (2.4) cannot be split into separately consistent $\mathrm{U}(1)$ and $\mathrm{SU}(n)$ bundles, but it could be separated into consistent $\mathrm{U}(1) / \mathbb{Z}_{n}$ and $\mathrm{SU}(n) / \mathbb{Z}_{n}$ bundles if there were no particles transforming under the center $\mathbb{Z}_{n}$ of $\mathrm{SU}(n)$. In physicist's language the latter bundle, although flat, carries a non-zero 't Hooft flux [31, which is responsible for the breaking of the observable gauge symmetry and the reduction of its rank from $n$ to 1 . The 't Hooft flux is an obstruction to " $n$-ality", or to "fundamental structure" of the $\mathrm{SU}(n) / \mathbb{Z}_{n}$ bundle, much like the obstruction to vector structure which is the subject of the present note.

Generalizing the above example, we can define an obstruction to "n-ality" for any $\mathrm{SU}(n) / \mathbb{Z}_{n}$ bundle $\widehat{V}$, whether flat or not. If this is part of a consistent $\mathrm{U}(n)$ bundle, then 
the obstruction can be related to the $\mathrm{U}(1)$ flux, encoded in the Wilson loop

$$
\mathcal{W}_{n}\left(\widehat{V}, T^{2}\right) \equiv \widehat{\mathcal{U}}\left(x^{9}+1\right) \widehat{\mathcal{U}}^{\dagger}\left(x^{9}\right)=e^{i \int_{T^{2}} \operatorname{tr} F / n}=e^{2 \pi i m / n} \in \mathbb{Z}_{n}
$$

The obstruction is thus determined by the number of D-particles modulo the number of D2-branes. Note that bundles with abelian transition functions and integer $m^{I}$ are also obstructed whenever $m \neq 0(\bmod n)$. Note also that when $n$ is not prime the obstruction may concern only a subgroup of the center $\mathbb{Z}_{n}$.

In slightly more mathematical terms, the internal gauge fields we are considering correspond to stable $\mathrm{U}(n)$ bundles on the torus. Stability here guarantees that the field strength of the associated connection is constant, i.e. a solution of the hermitian Yang-Mills equation. ${ }^{2}$ Given such a stable $\mathrm{U}(n)$ bundle $V$, then if $c_{1}(V)=m \in n \mathbb{Z}$ we can split off a line bundle $\mathcal{L}$ as $V=\widehat{V} \otimes \mathcal{L}$, where the structure group of $\widehat{V}$ is now $\mathrm{SU}(n)$. It is known that stable $\mathrm{SU}(n)$ bundles on a torus split into the direct sum of $n$ line bundles, $\widehat{V}=\bigoplus_{i} \mathcal{L}_{i}$ (see e.g. [32]). As a result of this splitting, the rank of the visible gauge group is not reduced, and we are left with a $\mathrm{U}(1)^{n}$ gauge theory. The above splitting does not, however, occur for stable $\mathrm{U}(n)$ bundles with $c_{1}(V)$ not a multiple of $n$, or for stable $\mathrm{SU}(n) / \mathbb{Z}_{n}$ bundles. Such bundles can, however, be always obtained by deforming the direct sum of line bundles into a non-trivial extension. In the type IIA language, a piecewise-linear D-string passing at each step through a node of the compactification lattice can be deformed, after enlarging the structure group, to a linear D-string of minimal length. This is the meaning of switching on non-trivial 't Hooft flux.

In what follows we will be interested in the particular case of $n=32$ D-branes, with first Chern class $m=-16$. From equation (2.7) we conclude that the D-branes carry an $\mathrm{SU}(32) / \mathbb{Z}_{2}$ bundle whose lift to a full SU(32) bundle is obstructed. There exist two simple choices for such an obstructed bundle: (i) a flat bundle with $\mathbb{Z}_{2}$ 't Hooft fluxes which corresponds to joining the dual D-strings in pairs, leading to a reduction of the rank from 32 to 16; or (ii) a bundle with half-integer magnetic fields which, when combined with the $\mathrm{U}(1)$ flux, make all the $f^{I} / 2 \pi$ integer. The rank in this case is not reduced. An example that illustrates this second option is the abelian $\mathrm{U}(1)^{32}$ bundle

$$
\begin{aligned}
\frac{F_{89}}{2 \pi} & =\operatorname{diag}[0, \ldots 0,-1, \ldots-1] \\
& =-\frac{1}{2} \operatorname{diag}[1, \ldots 1,1, \ldots 1]+\frac{1}{2} \operatorname{diag}[1, \ldots 1,-1, \ldots-1],
\end{aligned}
$$

where in the first line there are 16 zeros and 16 minus ones. Note that extracting the diagonal U(1) left us with "half-integer" magnetic fields in the remaining $\mathrm{SU}(32) / \mathbb{Z}_{2}$ bundle. Mixed configurations, with both half-integer magnetic fields and $\mathbb{Z}_{2}$ 't Hooft fluxes, are also possible as we discuss later.

\subsection{Relation to the $B$-flux}

The above considerations made no assumptions about the closed-string moduli. The NSNS background $B_{89}$ does, however, affect the dynamics of the magnetized D-branes, as is

\footnotetext{
${ }^{2}$ On the T-dual IIA side the corresponding D-strings are linear and thus special Lagrangian.
} 
evident for instance from the fact that the U(1) magnetic field appears in the Dirac-BornInfeld action only through the invariant combination $\mathcal{F}=B \mathbf{1}+F$. This is invariant under the NS-NS gauge transformations

$$
B_{\mu \nu} \rightarrow B_{\mu \nu}+\partial_{\mu} \Lambda_{\nu}-\partial_{\nu} \Lambda_{\mu} \quad \text { and } \quad A_{\mu} \rightarrow A_{\mu}-\Lambda_{\mu} \mathbf{1}
$$

where the one-form $\Lambda$ defines a U(1) bundle over $T^{2}$ (we use here the convention $2 \pi \alpha^{\prime}=1$ ). Large gauge transformations change, as is well-known, the number of D0-branes. Choosing, for instance, $\Lambda=2 \pi x^{8} d x^{9}$ transforms $f^{I} \rightarrow f^{I}-2 \pi$ and hence $m \rightarrow m-n$. The first Chern class $c_{1}(F)$ defines therefore a quantized but not gauge-invariant charge.

A "physical" D0-brane charge, which is gauge-invariant but not quantized, ${ }^{3}$ can be defined as the first Chern class of the bundle $\mathcal{V}$ with field strength $\mathcal{F}$,

$$
q \equiv \frac{1}{2 \pi} \int_{T^{2}} \operatorname{tr} \mathcal{F}_{89}=m+\frac{n}{2 \pi} \int_{T^{2}} B_{89} .
$$

Notice that the background $B$ field induces (fractional) D0 charge on the D2-branes, in the same way as the Yang-Mills $\theta$-angle induces electric charge on magnetic monopoles [39]. Suppose now that we insist that the physical D0-brane charge vanish. From equations (2.10) and (2.7) we then conclude that $\mathcal{V}$ is identified with the $\mathrm{SU}(n) / \mathbb{Z}_{n}$ bundle $\widehat{V}$ (put differently the $B$ field cancels the diagonal-U(1) part of $F$ ), and that

$$
\mathcal{W}_{n}\left(\widehat{V}, T^{2}\right)=e^{-i \int_{T^{2}} B} .
$$

Thus the obstruction to " $n$-ality" of the $\mathrm{SU}(n) / \mathbb{Z}_{n}$ bundle is determined by the flux of $B$, if one insists that $q=0$. As we will argue momentarily, this latter condition is automatic in the type I theory where the D2-branes are replaced by D9-branes and there is no R-R 8-form to which D7-brane charge can couple. This reasoning establishes the formula (1.1) of [5].

Before including orientifolds, let us translate these statements into the more intuitive T-dual language. Let the Kähler and complex structure moduli of the original 2-torus, which we take for simplicity orthogonal, be

$$
T=\frac{1}{2 \pi}\left(-B_{89}+i \ell_{8} \ell_{9}\right) \quad \text { and } \quad U=i \frac{\ell_{8}}{\ell_{9}},
$$

where $\ell_{j}$ are the circumferences of the two circles. A T-duality along $x^{9}$ exchanges $T$ with $U$, so that for non-zero $B$-field the dual torus is a tilted torus. The large gauge transformations (2.9) correspond to the complex structure transformations $\tilde{U} \rightarrow \tilde{U}-$ 1 , which shift the D-string winding numbers appropriately, $(n, m) \rightarrow(n, m-n)$. The obstruction to $n$-ality, determined by $m(\bmod n)$, is not affected by this shift. The physical D0-brane charge, $q$, measures the (net oriented) projection of the D-string on the imaginary axis of the complex plane with coordinate

$$
z \equiv i\left(\tilde{x}^{9}-\tilde{U} x^{8}\right)
$$

\footnotetext{
${ }^{3}$ The different notions of charge are even subtler in the general case where the NS-NS 3-form $H=d B$ does not vanish. For a discussion see references [33- 38].
} 

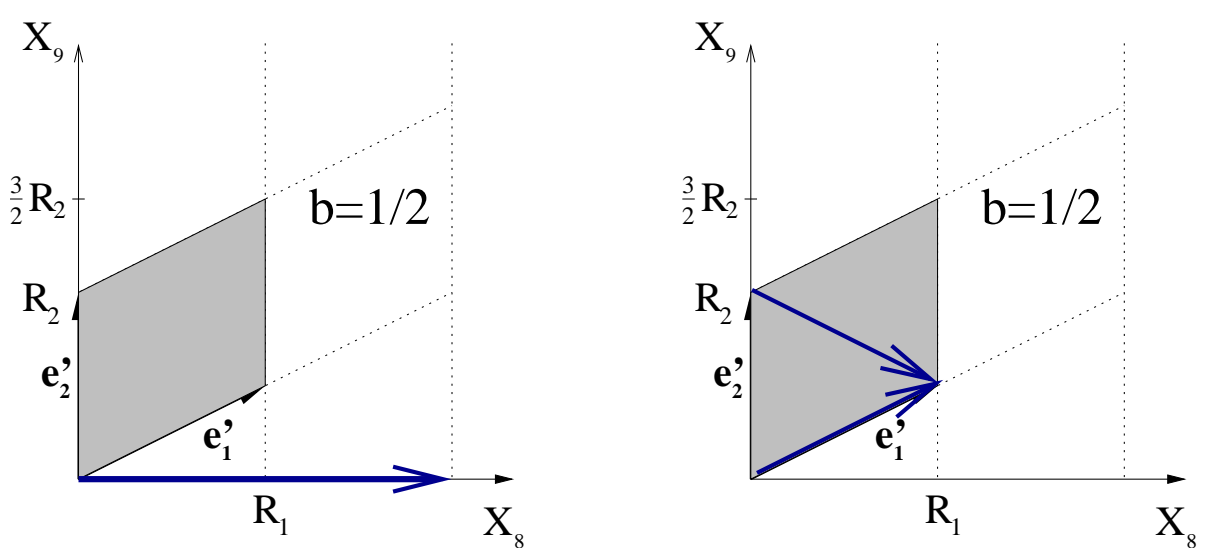

Figure 2: Two configurations of D8-branes canceling the tadpole with the branes indicated by the blue arrows.

One can easily check that the D-string with winding numbers $(n, m)$ is parallel to the real- $z$ axis when $q$ vanishes. Roughly speaking, the rotation of the D-string undoes the torus tilt in this case. This (minimal-length) D-string is dual to a stack of D2-branes carrying a flat $\mathrm{SU}(n) / \mathbb{Z}_{n}$ bundle.

\subsection{Including the orientifold}

We are now ready to consider the modding out by $\Omega \mathcal{R}$, where $\Omega$ is the reflection of the worldsheet coordinate $\sigma$, and $\mathcal{R}$ is a $\mathbb{Z}_{2}$ transformation of the (generalized) target spacetime. In the type IIA theory $\mathcal{R}$ flips the orientation of the 2 -torus, so $\tilde{T}^{2} / \mathcal{R}$ is one of the three open and/or unoriented genus-1 surfaces: the annulus, the Klein bottle or the Möbius strip. ${ }^{4}$ The first two have a purely-imaginary complex structure, whereas the third has, in our conventions, $\operatorname{Re} \tilde{U}=-1 / 2$. From eq. (2.12) we see that its T-dual configuration has $B_{89}=\pi$, so this is the case of interest to us here. The action of $\mathcal{R}$ in this case is

$$
\mathcal{R} z=\bar{z} \Longleftrightarrow \mathcal{R}\left(x^{8}, \tilde{x}^{9}\right)=\left(x^{8},-\tilde{x}^{9}-x^{8}\right) .
$$

The fixed-point surface, $x^{8}=-2 \tilde{x}^{9}$, is an orientifold 8-plane along the connected boundary of the Möbius strip. It has winding numbers $(2,-1)$ on the doubling torus, as illustrated in figure 2. To cancel its R-R 9-form charge we need, therefore, to introduce D8-branes with total winding numbers $(32,-16)$. Allowed configurations must be invariant under the action of $\Omega \mathcal{R}$ which is modded out.

Figure 2 shows two simple configurations that do the job. The first configuration has 16 D8-branes along the boundary of the Möbius strip, i.e. with winding numbers $(2,-1)$ for each D8-brane. Because they sit on top of the orientifold, these D8-branes and their $\mathcal{R}$-images coincide. This is the supersymmetric vacuum, discussed in refs. [18, 2, 3], which is dual to the heterotic CHL models 40 . It corresponds to a flat $\operatorname{Spin}(32) / \mathbb{Z}_{2}$ bundle with non-commuting Wilson lines [2, 3]

$$
W_{1} W_{2}=e^{-i \int_{T^{2}} B} W_{2} W_{1} .
$$

\footnotetext{
${ }^{4}$ Because of the action of $\Omega$ these surfaces should not be literally thought of as the compactification space.
} 
The second configuration in 2, on the other hand, has 16 D8-branes plus their mirror images under $\mathcal{R}$, with winding numbers respectively $(1,0)$ and $(1,-1)$. This is the configuration on the first of the three tori of model $\mathrm{C}$ [19]. Standing on its own configuration (ii) is actually unstable, because the $(1,0)$ and $(1,-1)$ mirror pairs can recombine to form $(2,-1)$ branes. $^{5}$ Model C "cures" this instability by exploiting the existence of the other compactified dimensions.

The T-duals to the configurations of figure 2 are precisely the gauge bundles described at the end of subsection 2.1. We can make this identification more explicit by looking at the action of $\Omega \mathcal{R}$ on the matrix-valued field $Y^{9}\left(x^{8}\right)$, which describes (in static gauge) the transverse position of the D8-branes. Consistently with the geometric action (2.14) this reads

$$
\Omega \mathcal{R}\left(Y^{9}\right)=-\gamma_{\Omega}\left(Y^{9}\right)^{t} \gamma_{\Omega}^{-1}-x^{8} \mathbf{1}
$$

where we choose (without loss of generality) the Chan-Paton basis so that

$$
\gamma_{\Omega}=\left(\begin{array}{cc}
0 & \mathbf{1}_{16 \times 16} \\
\mathbf{1}_{16 \times 16} & 0
\end{array}\right)
$$

The general solution to the above condition is of the form

$$
Y^{9}=-\frac{1}{2} x^{8} \mathbf{1}+\widehat{Y}^{9}
$$

where $\widehat{Y}^{9}$ takes values in the Lie algebra of $\mathrm{SO}(32)$. Using the T-duality dictionary, $A_{9}=2 \pi Y^{9}$, one can now easily check that the gauge bundles of subsection 2.1 are indeed T-dual to those of figure 2 .

Note that although the $\mathrm{U}(1) \subset \mathrm{U}(32)$ gauge field is projected out of the spectrum of the orientifold theory, a discrete background for it actually survives. Its role is to cancel the discrete $B$ flux so that $\operatorname{tr} \mathcal{F}=0$. Some of the confusion in the literature is due to a lack of appreciation of this subtle point. The obstruction to vector structure of the split-off bundle is related to this discrete $\mathrm{U}(1)$ flux and hence, by the previous argument, to the discrete $B$ modulus [18]. Furthermore, the physical D7-brane charge is automatically zero, consistently with the fact that the type I theory has no R-R 8-form to which this charge could couple.

Now consider a general configuration of D-branes which is easier to describe in the type IIA language. One accounts for both 't Hooft fluxes and magnetic fields by considering stacks of D8-branes with arbitrary integer winding numbers. Let the $a$-th stack have $N_{a}$ D8-branes with (relatively-prime) winding numbers $\left(n_{a}, m_{a}\right)$. For every stack we must also include the mirror stack with winding numbers $\left(n_{a},-m_{a}-n_{a}\right)$. Stacks with $n_{a}=-2 m_{a}$ can, a priori, be their own image. The cancellation of $\mathrm{R}-\mathrm{R}$ charge requires that $n \equiv \sum_{a} N_{a} n_{a}=$ 32. In the T-dual language the $a$-th stack carries a $\mathrm{U}\left(n_{a} N_{a}\right)$ gauge bundle which has 't Hooft flux that breaks the symmetry to $\mathrm{U}\left(N_{a}\right)$, and a $\mathrm{U}(1)$ magnetic field equal to

$$
F_{89}^{a}=\frac{2 \pi m_{a}}{n_{a}} \mathbf{1}_{N_{a} \times N_{a}} \otimes \mathbf{1}_{n_{a} \times n_{a}} .
$$

\footnotetext{
${ }^{5}$ But it is interesting to observe that this is not allowed for a single mirror pair of D-branes.
} 
Our normalization is such that fundamental-string endpoints have charge \pm 1 . As one can easily check, reflection symmetry fixes automatically the first Chern class of the complete $\mathrm{U}(32)$ bundle, as advertised

$$
m \equiv \frac{1}{2 \pi} \sum_{a} \operatorname{tr} F_{89}^{a}=\sum_{a} N_{a} m_{a}=-16
$$

Since $m / n=-1 / 2$, separating the diagonal $\mathrm{U}(1)$ gives an $\mathrm{SU}(32) / \mathbb{Z}_{2}$ bundle $\widehat{V}$, whose lift to an $\mathrm{SU}(32)$ bundle is obstructed. Now in accordance with the type I symmetry, the structure group of the bundle should actually be $\mathrm{SO}(32) / \mathbb{Z}_{2} \cdot{ }^{6}$ The reduction is automatic if none of the D8-branes is its own image. In this case the full transition matrices have the block-diagonal form

$$
\widehat{\mathcal{O}}=\left(\begin{array}{cc}
\mathcal{U} & 0 \\
0 & \mathcal{U}^{*}
\end{array}\right)
$$

and take values in $\mathrm{SO}(32)$ defined as the subgroup of matrices that obey the reality condition $\widehat{\mathcal{O}}^{*}=\gamma_{\Omega} \widehat{\mathcal{O}} \gamma_{\Omega}^{-1}$ and have determinant 1 . Such bundles can be thus written as the sum of two conjugate $\mathrm{U}(16) / \mathbb{Z}_{2}$ bundles, $\widehat{V}=\widehat{W} \oplus \widehat{W}^{\vee}$. The story is subtler for D8-branes which are their own image, and which are hence stuck to the orientifold plane. The elementary "stuck" D8-brane has winding numbers $(2,-1)$ and $\widehat{Y}^{9}=0$. From eqs. (2.5) and (2.6) we see that the corresponding $\mathrm{U}(2)$ transition function for this D-brane reads:

$$
\mathcal{U} \equiv e^{-i \pi x^{9}} \mathcal{O} \quad \text { with } \quad \mathcal{O}=e^{-i \pi x^{9} \sigma_{3}} \sigma_{1}
$$

where $\sigma_{i}$ are the usual Pauli matrices. The global phase in the split-off bundle was here fixed by imposing the reality condition $\mathcal{O}^{*}=\sigma_{1} \mathcal{O} \sigma_{1}$, where we think of $\mathcal{O}$ as occupying the $2 \times 2$ block in the center of the full $O(32)$ matrix. Since $\operatorname{det} \mathcal{O}=-1$, if one insists that the full structure group be $\mathrm{SO}(32) / \mathbb{Z}_{2}$ then stuck D8-branes are not permitted. An even number of $(2,-1)$ D8-branes can, on the other hand, be always combined in mirror pairs.

This obstruction to the existence of a good $\mathrm{SO}(32) / \mathbb{Z}_{2}$ bundle is described by an element of a mod 2 cohomology, the first Stiefel-Whitney class $w_{1} \in H^{1}\left(X, \mathbb{Z}_{2}\right)$. It is the same obstruction that prevents a pair of $(1,0)$ and $(1,-1)$ D8-branes to merge into a $(2,-1)$ brane, as we previously noted. The gauge group of perturbative type I theory is, as a matter of fact, $O(32) / \mathbb{Z}_{2}$. It is non-perturbative consistency (see the following section) which requires that $w_{1}=0$, and forbids ${ }^{7}$ the existence of "stuck" D8-branes and of $\mathrm{SO}(2 k+1)$ gauge groups in $T^{2}$ compactifications 13 . In the heterotic theory the vanishing of $w_{1}$ is a perturbative requirement, which follows from multiloop modular invariance [41]. A geometric interpretation of an analogous obstruction for D7-branes stuck on an O7-plane in IIB orientifolds has recently appeared in [42].

\footnotetext{
${ }^{6}$ We will discuss the requirement of being spin-liftable to $\operatorname{Spin}(32) / \mathbb{Z}_{2}$ in section 3.1 .

${ }^{7} \mathrm{SO}(2 k+1)$ factors in the gauge group are possible in lower dimension in the presence of 'exotic' $\widetilde{\Omega}$-planes with (quantized) R-R fluxes [14.
} 


\section{Toroidal $4 \mathrm{~d}$ orientifolds and the model $\mathrm{C}$}

Now we move on to more realistic backgrounds, obtained by compactification of type I theory on a six-torus with a non-flat $\mathrm{SO}(32) / \mathbb{Z}_{2}$ bundle [19]. These backgrounds are Tdual to (non-supersymmetric) intersecting D6-brane models [20, 22]. We restrict attention to factorizable tori, $T^{6}=T^{2} \times T^{2} \times T^{2}$. For a discussion of the non-factorizable case see the recent papers [43, 44].

\subsection{Consistency conditions for $T^{6} / \Omega \mathcal{R}$ orientifolds}

Let $\left(n_{a}^{i}, m_{a}^{i}\right)$ be the integer wrapping numbers of the ath stack of D6-branes on the $i$ th torus, and $\left(n_{a}^{i},-m_{a}^{i}-2 b^{i} n_{a}^{i}\right)$ the wrapping numbers of the mirror stack. We have defined here $b^{i}=1 / 2$ or 0 , according to whether the $i$ th torus is a tilted torus or not. In the type-I language this corresponds to a $B$-flux equal, respectively, to $\pi$ or to 0. Following reference [22] it is also convenient to introduce the shifted or "effective" wrapping numbers

$$
\hat{m}_{a}^{i} \equiv m_{a}^{i}+b^{i} n_{a}^{i}, \quad \text { so that } \quad \mathcal{R}\left(n_{a}^{i}, \hat{m}_{a}^{i}\right)=\left(n_{a}^{i},-\hat{m}_{a}^{i}\right) \quad \forall b^{i} .
$$

The $\hat{m}_{a}^{i}$ can be considered as wrapping numbers along the T-dualized directions of the three rectangular tori, or as magnetic fields from which the diagonal U(1) was stripped-off. Note that the $B$-fluxes enter through the quantization conditions,

$$
\hat{m}_{a}^{i}=m_{a}^{i}+b^{i} n_{a}^{i}, \quad \text { where } \quad n_{a}^{i}, m_{a}^{i} \in \mathbb{Z} .
$$

For tori with $b^{i}=\frac{1}{2}$ the $\hat{m}_{a}^{i}$ must be integer if $n_{a}^{i}$ is even, and half-integer if $n_{a}^{i}$ is odd, while when $b^{i}=0$ the $\hat{m}_{a}^{i}$ are always integer. The definition (3.1) makes it possible to treat both untilted and tilted tori in a unified way.

Tadpole cancellation for the R-R 7 -forms gives one condition for each independent 3 -cycle. On $T^{6}$ there are a priori 203 -cycles, but only 4 of them are even under the $\mathcal{R}$ reflection, $z^{i} \rightarrow \bar{z}^{i}$ for all $i$. One of them is the orientifold 3-cycle, and the other three share with the orientifold one dimension. The corresponding tadpole conditions (counting branes and their images separately) read [20, 22]

$$
\begin{array}{r}
\sum_{a=1}^{2 K} N_{a} n_{a}^{1} n_{a}^{2} n_{a}^{3}=32, \\
\sum_{a=1}^{2 K} N_{a} n_{a}^{1} \hat{m}_{a}^{2} \hat{m}_{a}^{3}=\sum_{a=1}^{2 K} N_{a} n_{a}^{2} \hat{m}_{a}^{1} \hat{m}_{a}^{3}=\sum_{a=1}^{2 K} N_{a} n_{a}^{3} \hat{m}_{a}^{1} \hat{m}_{a}^{2}=0 .
\end{array}
$$

Thanks to reflection symmetry, tadpole cancellation for the remaining odd cycles is automatic. Note that if the $n_{a}^{i}$ are positive, i.e. if there are no anti-D6-branes, then maximal rank is achieved when $n_{a}^{i}=1$ for all stacks and for all tori. Any $n_{a}^{i}>1$ implies a corresponding reduction of the rank.

Using the dictionary of the previous section, it is easy to translate the above statements into the language of magnetized D9-branes. The first of the conditions (3.3) fixes the total 
number of D9-branes, while the other three ensure that the second Chern class of the $\mathrm{SO}(32) / \mathbb{Z}_{2}$ bundle on them vanishes:

$$
\int_{T_{i}^{2} \times T_{j}^{2}} \operatorname{tr}\left(F^{i} \wedge F^{j}\right)=0 \quad \forall i, j=1,2,3
$$

These are precisely the conditions for cancellation of D5-brane charge. The total gauge bundle has structure group $\otimes_{a=1}^{K} \mathrm{SO}\left(2 n_{a}\right) / \mathbb{Z}_{2}$, where $n_{a} \equiv n_{a}^{1} n_{a}^{2} n_{a}^{3}$ and we have put here stacks and image stacks in a single factor. The wrapping numbers $\left(n_{a}^{i}, \hat{m}_{a}^{i}\right)$ describe the 't Hooft flux and magnetic fields of each separate $\mathrm{SO}\left(2 n_{a}\right) / \mathbb{Z}_{2}$ bundle. Each of these bundles has an obstruction to vector structure on the $i$ th torus whenever $b^{i} \neq 0$.

Apart from R-R tadpole cancellation, additional conditions come from the by now recurrent observation that, while the perturbative gauge group is $O(32) / \mathbb{Z}_{2}$, the full nonperturbative symmetry 45$]$ of type I theory is $\operatorname{Spin}(32) / \mathbb{Z}_{2}$. Recall that $\operatorname{Spin}(32)$ has four conjugacy classes: $O, V, S$, and $C$, corresponding respectively to the adjoint, the vector, the positive-chirality and the negative-chirality spinors. In terms of 16-dimensional root/weight vectors these are described as follows:

$$
\begin{array}{ccc}
O: & (0, \ldots, 0, \pm 1,0, \ldots, 0, \pm 1,0, \ldots, 0) & \\
V: & (0, \ldots, 0, \pm 1,0, \ldots, 0) & \\
S: & \left( \pm \frac{1}{2}, \pm \frac{1}{2}, \ldots, \pm \frac{1}{2}\right): & \text { even number of }+
\end{array}
$$$$
C: \quad\left( \pm \frac{1}{2}, \pm \frac{1}{2}, \ldots, \pm \frac{1}{2}\right): \quad \text { odd number of }+ \text {. }
$$

To obtain $\mathrm{SO}(32)$ from $\operatorname{Spin}(32)$ one projects out the spinor representations $S$ and $C$, while keeping the adjoint and the vector. Keeping only the adjoint gives the symmetry $O(32) / \mathbb{Z}_{2}$. By contrast, $\operatorname{Spin}(32) / \mathbb{Z}_{2}$ keeps the adjoint $O$ and the positive chirality spinor representation $S$, while projecting out the vector representation and the other spinor.

The spinor representation $S$ arises in type I theory via D-particle states which are nonBPS yet stable in $D=10$ and become BPS upon toroidal compactification [46]. These are dual to massive states of the heterotic string. Because states with the 'wrong' chirality $C$ do not exist, the parity transformation is not defined. Alternatively, the reduction of $O(32)$ to $\mathrm{SO}(32)$ can be traced to the existence of non-BPS D-instantons [47]. One immediate consequence, encountered already in subsection 2.3, is that the first Stiefel-Whitney class, $w_{1}$, must vanish. As explained there, the complete $\mathrm{SO}(32) / \mathbb{Z}_{2}$ bundle can then be written as the sum of two conjugate $\mathrm{U}(16) / \mathbb{Z}_{2}$ bundles, $\widehat{V}=\widehat{W} \oplus \widehat{W}^{\vee}$.

Such bundles are spin-liftable if the standard Dirac-quantization condition for charges in $S$ is satisfied, i.e. if the first Chern class of $\widehat{W}$ is even. Explicitly,

$$
\int_{T_{(3)}^{2}} c_{1}(\widehat{W})=\sum_{a=1}^{K} N_{a} n_{a}^{1} n_{a}^{2} \hat{m}_{a}^{3} \in 2 \mathbb{Z},
$$

and similarly for the other two tori. Note that the sum here runs over all D-brane stacks, but not over their mirrors. Condition (3.5) is known as the vanishing of the second StiefelWhitney class which, like the obstruction to vector structure, is an element of a mod 2 cohomology, $w_{2}(\widehat{V})=c_{1}(\widehat{W}) \bmod 2 \in H^{2}\left(X, \mathbb{Z}_{2}\right)$, which obstructs the existence of spin structure. It can be formulated as the requirement of cancellation of K-theory charge 48, 47] 
which is stronger than the mere cancellation of R-R tadpoles. Violation of (3.5) manifests itself also in the form of global $\mathrm{SU}(2)$ anomalies 49] in the world-volume theory of probe D5-branes [50].

\subsection{Three generations and the model $\mathrm{C}$}

For any solution of the consistency conditions (3.3), subject to the quantization rules (3.2) and (3.5), some of the most interesting observables are the intersection numbers of stacks of D6-branes:

$$
I_{a b}=\prod_{i=1}^{3}\left(n_{a}^{i} \hat{m}_{b}^{i}-n_{b}^{i} \hat{m}_{a}^{i}\right) .
$$

These determine the chiral spectrum in the effective four-dimensional theory, and in particular the number of Standard-Model or GUT generations in (semi)realistic models of this kind. Note that the intersection numbers $I_{a b}$ are not affected by the shift (3.1), which is why the integer winding numbers $m_{a}^{i}$ could be replaced by $\hat{m}_{a}^{i}$ in the above expression. Recall also that in deriving the chiral spectrum $[a b]$ and $\left[a b^{\prime}\right]$, where $b^{\prime}$ is the mirror of the stack $b$, should be considered separately.

Let us review now (and sharpen a little) the argument [22] which shows that 3generation toroidal models can only exist when one or more of the tori are tilted. We focus on the left-handed quarks, which correspond to open strings stretching between the color and weak-isospin stacks of D-branes (denoted here by the labels $c$ and $w$ ). To get 3 generations we need that $I_{\mathrm{cw}}+I_{c w^{\prime}}=3$. Generic models have $I_{\mathrm{cw}}=3$ and $I_{c w^{\prime}}=0$, but because the 2 and $\overline{2}$ representations are equivalent we only require the above weaker condition. Now the mirror to the weak-isospin stack is obtained by flipping the sign of the $\hat{m}_{w}^{i}$, so that

$$
I_{\mathrm{cw}}+I_{c w^{\prime}}=-2 \prod_{i=1}^{3}\left(n_{w}^{i} \hat{m}_{c}^{i}\right)-2\left(n_{c}^{1} \hat{m}_{w}^{1} n_{c}^{2} \hat{m}_{w}^{2} n_{w}^{3} \hat{m}_{c}^{3}+\text { cyclic }\right) .
$$

This can be odd only if some of the effective wrapping numbers are half-integers, which implies in turn that at least one of the tori must be tilted. Bundles without vector structure are thus unavoidable in all realistic toroidal-orientifold models.

One of the nice features of the 3-family Grand-Unified model $\mathrm{C}$ is that it is obtained with a very simple choice for the $\mathrm{SO}(32) / \mathbb{Z}_{2}$ bundle [19]. The choice is exhibited in table 1.

From our previous discussion it should be clear that the bundle admits no vector structure, and requires $b^{i}=1 / 2$ on all three tori. If instead the $b^{i}$ were zero, we would need to multiply the wrapping numbers $\hat{m}_{a}^{i}$ by a factor 2 , thereby increasing to 24 the number of families [20]. The construction of this model predated the discovery of the nonperturbative structure of type I theory [45], so the vanishing of the second Stiefel-Whitney class, eqs. (3.5), was not checked at that time. One can, however, verify that not only the tadpole conditions (3.3), but also eqs. (3.5) are satisfied, so that the bundle $\mathrm{C}$ can be lifted to a fully-consistent $\operatorname{Spin}(32) / \mathbb{Z}_{2}$ bundle.

The Standard-Model gauge group in model C is unified in the SU(5) group on the first stack of D6-branes. There is also a horizontal $\mathrm{U}(3)$ symmetry and a $\mathrm{U}(4) \times \tilde{\mathrm{U}}(4)$ hidden 


\begin{tabular}{|c|r|r|r|r|}
\hline stack & $\mathrm{U}(5)$ & $\mathrm{U}(3)$ & $\mathrm{U}(4)$ & $\tilde{\mathrm{U}}(4)$ \\
\hline$\left(n_{1}, \hat{m}_{1}\right)$ & $\left(1, \frac{3}{2}\right)$ & $\left(1,-\frac{5}{2}\right)$ & $\left(1, \frac{1}{2}\right)$ & $\left(1,-\frac{1}{2}\right)$ \\
\hline$\left(n_{2}, \hat{m}_{2}\right)$ & $\left(1, \frac{1}{2}\right)$ & $\left(1, \frac{1}{2}\right)$ & $\left(1,-\frac{1}{2}\right)$ & $\left(1,-\frac{1}{2}\right)$ \\
\hline$\left(n_{3}, \hat{m}_{3}\right)$ & $\left(1, \frac{1}{2}\right)$ & $\left(1, \frac{1}{2}\right)$ & $\left(1, \frac{1}{2}\right)$ & $\left(1, \frac{1}{2}\right)$ \\
\hline
\end{tabular}

Table 1: The wrapping numbers of model C.

sector. One can easily check that $I_{53}=-1$ and $I_{55^{\prime}}=3$, giving three generations in the 10 and $\overline{5}$ representations of $\mathrm{SU}(5)$. There is no chiral matter from strings between the hidden and observable stacks of D6-branes. The model is non-supersymmetric but free of tachyons, in appropriate regions of parameter space, and it has the necessary scalar fields for GUT, electroweak and horizontal-symmetry breaking [19]. The pattern of supersymmetry breaking is also rather interesting: the gauge sector is maximally-supersymmetric at tree level, and the breaking in the chiral-matter sector is tunable. The split-supersymmetry scenario [51, 22] can be thus implemented in this model naturally (bearing in mind the usual problems of vacuum stability). A simple variant of model $\mathrm{C}$ has been, in fact, analyzed in this spirit in ref. [53]. For other unified intersecting D-brane models see also [54-59].

\subsection{Euclidean D1-brane instantons}

In subsection 2.3 we have discussed the origin of the obstruction to vector structure for the $\operatorname{Spin}(32) / \mathbb{Z}_{2}$ bundle on the type I D9-branes. It is easy to extend this argument to Euclidean trajectories of D-strings (or instantonic E1-branes) that wrap an orientifolded two-torus with $B$-flux. The logic is the same as before: given $n$ branes of the above kind, we should look for $\mathrm{U}(n)$ gauge bundles, $A^{9}\left(x^{8}\right)$, that survive the twisted orientifold projection

$$
\Omega \mathcal{R}\left(A^{9}\right)= \pm \gamma_{\Omega}\left(A^{9}\right)^{t} \gamma_{\Omega}^{-1}-2 \pi x^{8} \mathbf{1}
$$

The sign here is + for the D5-branes and - for the E1-instantons, for reasons explained clearly in references [60, 61]. As in subsection 2.3, the general solution of the above condition is a "half-integer" magnetic field in the overall $\mathrm{U}(1) \subset \mathrm{U}(n)$ factor, and an $O(n) / \mathbb{Z}_{2}$ or $\operatorname{Sp}(n) / \mathbb{Z}_{2}$ bundle without vector structure on the E1-brane, respectively on the D5-brane world-volume.

The D5-branes in toroidal orientifolds are special limits of gauge bundles on the D9branes, so we will not discuss them here further. Let us consider instead in more detail E1-instantons wrapping the $i$ th $T^{2}$ factor, for which $b^{i}=\frac{1}{2}$. In the type IIA language, the corresponding instantonic trajectories must wrap invariant 1-cycles of $T^{2} / \mathcal{R}$, and all such 
cycles have even winding number, $n=2 k$, in the $x^{8}$ direction. This means, when translated in the type I language, that only an even number of E1-branes, with a non-trivial $O(2 k) / \mathbb{Z}_{2}$ bundle on their worldvolume, can wrap the obstructed 2-cycle. Note that, in contrast to the D9-branes, the structure group for the E1-branes need not be reducible to $\mathrm{SO}(2 k) / \mathbb{Z}_{2}$. Note also that the complete gauge group for the combined system of D9- branes and E1-branes is $[\operatorname{Spin}(32) \times O(2 k)] / \mathbb{Z}_{2}$, where the "invisible" $\mathbb{Z}_{2}$ flips the sign of the vector representations of the two factor groups, thus leaving the bi-fundamental representation $(32,2 k)$ unchanged.

The fact that a single E1-instanton cannot wrap an obstructed 2-cycle has been observed previously in [62]. This does not, however, mean that the multiply-wrapped E1branes make no contributions to supersymmetry-protected quantities. Flatness of the Chan-Paton bundle is, of course, required for the instanton to be supersymmetric and thus have a chance of contributing to F-terms. Consider for example $n=2$ : a flat $O(2) / \mathbb{Z}_{2}$ bundle with 't Hooft flux is consistent with spacetime supersymmetry, and lifts half of the fermionic zero modes as is evident in the T-dual "long-string" picture. It should therefore contribute to the same quantities as the single $(n=1)$ E1-brane in compactifications without $B$-flux. In principle, with only the zero modes corresponding to Wilson lines along $T^{2}$, such an instanton could contribute to the gauge kinetic function on D9- or D5-branes. However, in the pure toroidal case considered here, there exist extra zero modes related to the transverse translations of the E1-instanton, so that such objects rather contribute to higher-derivative F-terms.

E1-instantons have attracted recently much attention, because they can generate phenomenologically desirable terms in the effective superpotential of type I models 66 66. Corrections to higher-derivative $\mathrm{F}$-terms for $N=1$ vacua were pioneered, in the context of heterotic worldsheet instantons, in [67] and discussed in the language of D-brane instantons in [68]. A nice guide for elucidating the type-I D-instanton calculus, in a simpler though less realistic setting, are the $F^{4}$ threshold corrections of maximally-supersymmetric, $N=4$ vacua [69-71]. The one-loop computation of these corrections on the heterotic side is exact [72], so the contributions of D-instantons are known. By comparing threshold corrections in Type I models with non-zero $B$ flux and in heterotic CHL models it is possible to verify that $\mathrm{D}$-instantons with even and odd $n$ correspond to different sectors of the freely acting orbifold [73]. Precise agreement between heterotic-worldsheet and E1-instanton corrections to 4 -hyperini Fermi couplings on $T^{4} / \mathbb{Z}_{2}$ has been recently demonstrated in 74 .

\section{Calabi-Yau compactifications of $\operatorname{Spin}(32) / \mathbb{Z}_{2}$ bundles with or without vector structure}

So far we have analyzed the simplest case of toroidal compactification. Much of the analysis carries, however, over to genuine Calabi-Yau spaces, with full SU(3) holonomy, as we will discuss in this section.

\section{1 $\operatorname{Spin}(32) / \mathbb{Z}_{2}$ gauge bundles}

Let us begin with type I compactifications on a general Calabi-Yau manifold $X$, which for conceptual simplicity we assume to be smooth. Through every 2-cycle $\gamma \in H_{2}(X, \mathbb{Z})$ we 
may turn on integer or half-integer $B$ flux, consistently with the $\Omega$ projection. These fluxes, and the corresponding discrete Kähler moduli $\operatorname{Re}\left(T_{i}\right)$, are described by an element $\mathcal{B} \in$ $H^{2}(X, \mathbb{Z} / 2)$, normalized so that $\mathcal{B}(\gamma) \equiv \int_{\gamma} B / 2 \pi$. Of course, only the mod2 cohomology $b \equiv[\mathcal{B}] \in H^{2}\left(X, \mathbb{Z}_{2}\right)$ describes physically-distinct vacua, so the number of inequivalent choices of discrete moduli is $2^{h_{11}(X)}$. We are interested in $\operatorname{Spin}(32) / \mathbb{Z}_{2}$ bundles on $X$. For background material on Type I compactifications with non-abelian vector bundles (but vanishing $B$-flux) we refer the reader to [75, 76].

As in the toroidal case, the $\operatorname{Spin}(32) / \mathbb{Z}_{2}$ bundle defining the type I model can be constructed by first considering a $\mathrm{U}(32)$ bundle $V$,

$$
V=\bigoplus_{a} V_{a}^{\oplus N_{a}} \oplus \bigoplus_{a}\left(V_{a}^{*}\right)^{\oplus N_{a}} .
$$

Here $V_{a}$ denotes a $\mathrm{U}\left(n_{a}\right)$ bundle with $c_{1}\left(V_{a}\right) \in H^{2}(X, \mathbb{Z})$, while the $*$ operation is defined by dualizing the bundle and then twisting it with a line bundle $\mathcal{N}$,

$$
V_{a}^{*}=V_{a}^{\vee} \otimes \mathcal{N} \quad \text { where } \quad c_{1}(\mathcal{N})=-2 \mathcal{B} \in H^{2}(X, \mathbb{Z}) .
$$

The twist bundle $\mathcal{N}$ accounts for the shift under the action of $\Omega$ which, in the toroidal case, was encoded in the transformation of wrapping numbers $(n, m) \rightarrow(n,-m-2 b n)$. The structure group $\mathrm{U}\left(n_{a}\right)$ of each $V_{a}$ is embedded diagonally into $\mathrm{U}\left(n_{a} N_{a}\right) \in \operatorname{Spin}(32) / \mathbb{Z}_{2}$ subject to the constraint $\sum n_{a} N_{a}=16$. The resulting four-dimensional gauge group is given by $\prod_{a} \mathrm{U}\left(N_{a}\right)$ (modulo massive $\mathrm{U}(1)$ factors) along the lines of [75, 76. It follows immediately from the above definitions that the total $\mathrm{U}(1)$ flux associated with $V$ equals -16,

$$
\int_{\gamma} c_{1}(V)=\frac{1}{2 \pi} \int_{\gamma} \operatorname{tr} F=-16
$$

for each two-cycle $\gamma \in H_{2}(X, \mathbb{Z})$ with half-integer $B$-flux. Exactly as in section 2, this guarantees vanishing D7-brane charge on the D9-branes.

The advantage of working with the $V_{a}$ is that they are conventional bundles with integer first Chern class. This comes at the cost of introducing the unusual orientifoldaction operator $*$, which is twisted by the appearance of the $B$-flux. Alternatively, we can use the conventional twist but work with bundles whose first Chern class can be half-integer. To this end we write

$$
V=\widehat{V} \otimes \mathcal{L}
$$

where the line bundle $\mathcal{L}$ is such that $c_{1}(\mathcal{L})=-\mathcal{B} \in H^{2}(X, \mathbb{Z} / 2)$. After splitting off the diagonal $\mathrm{U}(1)$ in this way, $\widehat{V}$ represents a $\operatorname{Spin}(32) / \mathbb{Z}_{2}$ bundle given by the direct sum of a $\mathrm{U}(16) / \mathbb{Z}_{2}$ bundle and its dual,

$$
\widehat{V}=\widehat{W} \oplus \widehat{W}^{\vee} \quad \text { with } \quad W=\bigoplus_{a} \widehat{V}_{a}^{\oplus N_{a}} .
$$

The generalization of the quantization condition eq. (3.2) reads

$$
c_{1}\left(\widehat{V}_{a}\right)+n_{a} b \in H^{2}(X, \mathbb{Z}) .
$$


For non-zero $b$ and odd rank $n_{a}$, in particular, the first Chern class $c_{1}\left(\widehat{V}_{a}\right)$ takes halfinteger values. This violates the Dirac quantization condition for the vector representation of $\mathrm{SO}(32)$, so that the bundle $\widehat{V}$ is an $\mathrm{SO}(32) / \mathbb{Z}_{2}$ bundle without vector structure.

For $\widehat{V}$ to be liftable to $\operatorname{Spin}(32) / \mathbb{Z}_{2}$, the bundle $\widehat{W}$ must furthermore satisfy the Dirac quantization condition with respect to the spin conjugacy class,

$$
c_{1}(\widehat{W})=\sum_{a} N_{a} \int_{\gamma} c_{1}\left(\widehat{V}_{a}\right) \in 2 \mathbb{Z} \quad \forall \gamma \in H_{2}(X, \mathbb{Z}) .
$$

This generalizes eq. (3.5). Finally, the tadpole cancellation condition for such Type I compactifications with D9-branes only is given by [75, 76]

$$
\operatorname{ch}_{2}(\widehat{W})+c_{2}\left(T_{X}\right)=0
$$

where $T_{X}$ denotes the tangent bundle of $X$.

In general, one can add D5-branes wrapping holomorphic curves in $X$ provided the total fivebrane class

$$
W_{5}=\operatorname{ch}_{2}(\widehat{W})+c_{2}\left(T_{X}\right)
$$

is effective. Recall that a 2 -cycle $\gamma$ with $\mathcal{B}(\gamma)=0$ can only be wrapped by $2 k$ fivebranes on $X$ (in the upstairs geometry). ${ }^{8}$ This yields gauge group $\operatorname{Sp}(k)$ in conventions where $\operatorname{Sp}(1)=\mathrm{SU}(2)$. For $\mathcal{B}(\gamma)=1 / 2$ we must invoke non-trivial 't Hooft flux which further breaks the gauge group on the five-branes. The minimal configuration on a smooth manifold now correpsonds to $2 \times 2$ fivebranes along $\gamma$ in the upstairs picture where each of the two pairs carries a non-trival $\mathrm{SU}(2) / \mathbb{Z}_{2}$ bundle. This yields again gauge group $\mathrm{Sp}(1)$ after modding out by the orientifold.

For E1-instantons, by contrast, if $\mathcal{B}(\gamma)=0$ the Chan-Paton group is $O(k)$ and no restrictions on $k$ arise. However, absence of vector structure along a 2-cycle due to $\mathcal{B}(\gamma)=\frac{1}{2}$ is an obstruction for the appearance of a single E1-instanton along $\gamma$ [62]. Here Dirac quantization would be violated for the charged zero modes between the E1-instanton and the magnetized D9-branes, which are discussed in Type I language in [63, 65, 666].

For E1-instantons to contribute to holomorphic quantities like the superpotential or the gauge kinetic functions, they must be of type $O(1)$, i.e. carry bundles satisfying $c_{1}\left(V_{a}\right)=0$. As a result, the quantization condition (4.6) can only be satisfied for even rank of the bundle. From this argument it seems to be possible that in fact the structure established in section 3.3 for E1's wrapping genus one curves can be generalized to for instance degree $k$ covers of isolated rational curves. Namely, for $k$ even the quantization condition is satisfied and a contribution to the superpotential seems to be possible. ${ }^{9}$

\footnotetext{
${ }^{8}$ The class $W_{5}$ in eq. (40) is the one after modding out by the orientifold action, i.e. it describes the set of $n$ five-branes.

${ }^{9}$ Note that the degree $k$ cover can be thought of as the image of the map $z \rightarrow z^{k}$ which has two $k$-fold branch cuts at $z=0, \infty$.
} 


\subsection{Smooth Calabi-Yau type IIA orientifolds}

Let us now discuss the mirror-dual side of type IIA orientifolds on general Calabi-Yau manifolds. We will identify, in particular, the discrete freedom in the choice of complex structure moduli which is dual to the choice of orientifolds with and without vector structure in type I.

Under mirror symmetry the pure world-sheet parity transformation $\Omega$ on a manifold $X$ is mapped to $\Omega \mathcal{R}(-1)^{F_{L}}$, where $\mathcal{R}$ denotes an anti-holomorphic involution on the miror dual Calabi-Yau manifold $\mathcal{W}$. It acts on the holomorphic $(3,0)$ form $\Omega_{3}$ and the Kähler two-form $J$ as

$$
\mathcal{R}: \Omega_{3} \rightarrow e^{2 i \theta} \bar{\Omega}_{3}, \quad \mathcal{R}: J \rightarrow-J .
$$

Without loss of generality we will set $\theta=0$ in what follows. The fixed point locus of $\mathcal{R}$ gives rise to an orientifold $O 6$-plane, whose tadpole is canceled by the introduction of D6-branes wrapping special Lagrangian 3-cycles on the Calabi-Yau manifold [77]. These D6-branes are wrapped around homology 3-cycles $\pi_{a}$.

Let us first review the case of mirror dual to Type I compactifications with zero $B$ field. The homology group $H_{3}(\mathcal{W}, \mathbb{Z})$ splits into an $\Omega \mathcal{R}$ even and odd part, $H_{3}(\mathcal{W}, \mathbb{Z})=$ $H_{3}^{+}(\mathcal{W}, \mathbb{Z}) \oplus H_{3}^{-}(\mathcal{W}, \mathbb{Z})[78]$. The even part contains real 3 -cycles and the odd part completely imaginary ones. Moreover, $\Omega \mathcal{R}$ exchanges the holomorphic and the antiholomorphic 3-forms, so that the volume form

$$
\operatorname{vol}(\mathcal{W})=\frac{i}{8} \Omega_{3} \wedge \bar{\Omega}_{3}
$$

is anti-invariant, i.e. $\Omega \mathcal{R}: \operatorname{vol}(\mathcal{W}) \rightarrow-\operatorname{vol}(\mathcal{W})$. Therefore, the only non-vanishing intersections are between 3 -cycles from $H_{3}^{+}(\mathcal{W}, \mathbb{Z})$ and $H_{3}^{-}(\mathcal{W}, \mathbb{Z})$.

One can always find a symplectic unimodular basis $\left(A_{I}, B_{I}\right)$ of $H_{3}(\mathcal{W}, \mathbb{Z}), I=$ $0, \ldots, h_{2,1}$, where we take $A_{0}, B_{i} \in H_{3}^{-}(\mathcal{W})$ and $B_{0}, A_{i} \in H_{3}^{+}(\mathcal{W})$ for $i=1, \ldots, h_{2,1}$. The intersection matrix for this choice of basis has the simple form $A_{I} \cap B_{J}=\delta_{I J}$ with all other intersection numbers vanishing. Note that this defines a Poincaré dual basis $\left(\alpha_{0}, \beta_{i}\right)$ of $H_{-}^{3}(\mathcal{W}, \mathbb{Z})$ and $\left(\beta_{0}, \alpha_{i}\right)$ of $H_{+}^{3}(\mathcal{W}, \mathbb{Z})$ such that

$$
\int_{A^{I}} \alpha_{J}=\delta_{I J}, \quad \int_{B^{I}} \beta_{J}=-\delta_{I J}, \quad\left(I, J=0, \ldots, h_{2,1}\right) .
$$

In this basis, the holomorphic three-form $\Omega_{3}$ is expanded as

$$
\Omega_{3}=\sum_{I} X_{I} \alpha_{I}-\sum_{J} F_{J} \beta_{J}
$$

in terms of the periods

$$
X_{I}=\int_{A_{I}} \Omega_{3}, \quad F_{J}=\int_{B_{J}} \Omega_{3} .
$$

Special geometry of the complex structure moduli space implies that the periods along $B_{I}$ can be expressed as derivatives of the prepotential $\mathcal{F}\left(U_{i}\right)$, where one defines the quotient of two periods

$$
U_{i}=\frac{X_{i}}{X_{0}}=\frac{\int_{A_{i}} \Omega_{3}}{\int_{A_{0}} \Omega_{3}} .
$$


In terms of these one has

$$
\frac{\partial \mathcal{F}}{\partial U_{i}}=\frac{\int_{B_{i}} \Omega_{3}}{\int_{A_{0}} \Omega_{3}}, \quad \mathcal{F}_{0} \equiv 2 \mathcal{F}-\sum_{i} U_{i} \frac{\partial \mathcal{F}}{\partial U_{i}}=\frac{\int_{B_{0}} \Omega_{3}}{\int_{A_{0}} \Omega_{3}} .
$$

The $U_{i}$ indeed transform under the orientifold action as $\Omega \mathcal{R}: U_{i} \rightarrow-\bar{U}_{i}$, and a consistent choice is $\operatorname{Re}\left(U_{i}\right)=0$.

As in 79] we expand the 3-cycles of the branes and the orientifold planes as

$$
\pi_{a}=\sum_{I=0}^{h_{2,1}}\left(q_{a, I} A_{I}-p_{a, I} B_{I}\right), \quad \pi_{\mathrm{O} 6}=\frac{1}{2}\left(L_{0} B_{0}+\sum_{i=1}^{h_{2,1}} L_{i} A_{i}\right) .
$$

The image brane has the expansion $\pi_{a}^{\prime}=-q_{a, 0} A_{0}-p_{a, 0} B_{0}+\sum_{i=1}^{h_{2,1}}\left(q_{a, i} A_{i}+p_{a, i} B_{i}\right)$. For a supersymmetric brane configuration the NS-NS tadpole cancellation condition takes the simple form

$$
-\sum_{a} N_{a} p_{a, 0} \mathcal{F}_{0}+\sum_{a, i} N_{a} q_{a, i} U_{i}=\left(L_{0} \mathcal{F}_{0}+\sum_{i} L_{i} U_{i}\right),
$$

where the terms of zero and second order in the $U_{i}$ cancel due to the image branes. Equation (4.18) encodes $h_{2,1}+1$ independent conditions on the wrapping numbers of the D6-branes.

Mirror duality maps this type IIA orientifold with intersecting D6-branes and O6-plane to type I= type IIB $/ \Omega$ compactifications with magnetized D9-branes. The type IIB Kähler moduli $T_{i}=-b_{i}+i J_{i}$ are defined by expanding $T=-\mathcal{B}+i J$ as $\sum_{i}\left(-b_{i}+i J_{i}\right) \omega_{i}=T^{i} \omega_{i}$, where $\omega_{i}$ denotes a basis of $H^{2}(X, \mathbb{Z})$. The mirror map exchanges the IIB moduli $T_{i}$ with the IIA complex structure moduli $U_{i}$ [80, 81]. The above choice $\operatorname{Re}\left(U_{i}\right)=0$ is obviously dual to $b_{i}=0$ on the type I side. Recall that in the type I case the possibility of half-integer NS-NS flux results from the periodic identification $\mathcal{B}(\gamma) \simeq \mathcal{B}(\gamma)+1$, which, together with $\mathcal{B} \rightarrow-\mathcal{B}$ under $\Omega$ allows for $\mathcal{B}(\gamma)=0$ or $\frac{1}{2}[18$. By mirror symmetry also the complex structure moduli $U_{i}$ enjoy a shift symmetry $U_{i} \simeq U_{i}-1$, so that the two discrete values $\operatorname{Re}\left(U_{i}\right)=0,-\frac{1}{2}$ are allowed. The value $\operatorname{Re}\left(U_{i}\right)=-\frac{1}{2}$ is the mirror dual of the type I orientifold without vector structure.

Note that the value $\operatorname{Re}\left(U_{i}\right)$ is still measured with respect to the old unimodular basis (4.12). In general, with the tilt in the complex structure, this basis ceases to take values in $H^{3}(\mathcal{W}, \mathbb{Z})$, but rather is defined only in $H^{3}(\mathcal{W}, \mathbb{Q})$. Of course one can now define a new basis of $H^{3}(\mathcal{W}, \mathbb{Z}),{ }^{10}$ but this basis does not split into even and odd parts under $\Omega \mathcal{R}$.

As on the torus one can choose to keep the nice transformation properties of the basis (4.12) and formally expand the three-cycles wrapped by the D-branes as in (4.17). The so-defined wrapping numbers are subject to certain constraints which ensure that the object $\pi$ is a bona fide cycle.

\footnotetext{
${ }^{10}$ For the toroidal case, this basis would be the one constructed from the fundamental cycles $e_{1}^{\prime}$ and $e_{2}^{\prime}$ in figure 2 .
} 
To find the correct description we use the fact that mirror symmetry exchanges the central charges of a B-type brane carrying a holomorphic bundle $V_{a}$ on a Calabi-Yau $X$ and the dual A-type brane wrapping the sLag $\pi_{a}$ on the mirror manifold $\mathcal{W}$. Recall that the central charges are defined as 82

$$
Z_{B}=\int_{X} e^{\mathcal{B}-i J} \operatorname{ch}\left(V_{a}\right) \sqrt{\operatorname{Td}\left(T_{X}\right)}, \quad Z_{A}=\int_{\pi_{a}} \Omega_{3} .
$$

The expression for $Z_{B}$ depends on the gauge field and the $B$-field only via the gauge invariant combination $\mathcal{F}=F+B .^{11}$

One now expands $Z_{B}$ and $Z_{A}$ along $H^{2}(X, \mathbb{Z})$ and $H^{3}(\mathcal{W})$ and uses the mirror map between $T_{i}$ and $U_{i}$ to express the 'wrapping numbers' $p_{I}$ and $q_{I}$ of the A-brane in terms of the topological data of the mirror dual bundle and the Todd class of $X$. To work this out explicitly requires the form of the prepotential $\mathcal{F}\left(U_{i}\right)$. As already anticipated one has here two choices: either work with the bundle $V_{a}$ as in eq. (4.19) with $c_{1}\left(V_{a}\right) \in H^{2}(X, \mathbb{Z})$. The corresponding wrapping numbers for the mirror dual A-brane will then be the ones with respect to the tilted basis taking values in $H_{3}(\mathcal{W}, \mathbb{Z})$. Alternatively one absorbs the $B$-field into the gauge bundle by writing

$$
Z_{B}=\int_{X} e^{-i J} \operatorname{ch}\left(\widehat{V}_{a}\right) \sqrt{\operatorname{Td}\left(T_{X}\right)}
$$

This will give us the effective fractional wrapping numbers along the unimodular basis valued in $H^{3}(\mathcal{W}, \mathbb{Q})$.

Let us treat the two cases in turn. First, one can expand $Z_{B}$ as 84

$$
Z_{B}=Q^{6}-T Q^{4}+\frac{1}{2} T^{2} Q^{2}-\frac{1}{6} T^{3} Q^{0}
$$

with

$$
\begin{aligned}
Q^{0} & =\operatorname{rk}(\mathcal{E}), \quad Q^{2}=c_{1}(\mathcal{E}), \quad Q^{4}=\operatorname{ch}_{2}(\mathcal{E})+\frac{r k(\mathcal{E})}{24} c_{2}\left(T_{X}\right) \\
Q^{6} & =\operatorname{ch}_{3}(\mathcal{E})+\frac{1}{24} c_{1}(\mathcal{E}) c_{2}\left(T_{X}\right) .
\end{aligned}
$$

where $\mathcal{E}$ collectively denotes the bundles $V_{a}$ or $\widehat{V}_{a}$, depending on whether we absorb the $B$-flux in the field $T=-b+i J$ or the gauge bundle.

The analogous expansion for $Z_{A}$ reads

$$
Z_{A}=\int_{\pi} \Omega_{3}=X_{0}\left(q_{0}+\sum_{i} q_{i} U_{i}-\sum_{i} p_{i} \frac{\partial \mathcal{F}}{\partial U_{i}}-p_{0} \mathcal{F}_{0}\right)
$$

Now one uses the mirror map to identify $T_{i}$ with $U_{i}$. In the large volume limit the prepotential $\mathcal{F}(T)$ takes the form

$$
\mathcal{F}(T)=-\frac{1}{6} T^{3}+\frac{1}{2} A T^{2}-\frac{1}{24} c_{2}\left(T_{X}\right) T .
$$

\footnotetext{
${ }^{11}$ To comply with the convention used in the discussion of toroidal models we have chosen $\mathcal{F}=F+B$ (as opposed to $F-B$ ) to be the gauge invariant combination. Consequently we have defined $Z_{B}$ in terms of $e^{\mathcal{B}-i J}$, rather than $e^{-(\mathcal{B}+i J)}$ as in 82 .
} 
This classical expression receives worldsheet instanton corrections away from the large volume limit. For a discussion of the terms linear and quadratic in $T$, which do not enter the tri-linear couplings, we refer e.g. to [83]. Using this result and comparing the expansions of $Z_{B}$ and $Z_{A}$ leads to 84

$$
\begin{aligned}
\left(p_{a}\right)_{0} & =\operatorname{rk}(\mathcal{E}), & \sum_{i}\left(p_{a}\right)_{i} \omega_{i} & =c_{1}(\mathcal{E}), \\
q_{0} & =\operatorname{ch}_{3}(\mathcal{E}), & \sum_{i}\left(q_{a}\right)_{i} \widetilde{\omega}_{i} & =-\left(\operatorname{ch}_{2}(\mathcal{E})+\frac{\operatorname{rk}(\mathcal{E})}{12} c_{2}\left(T_{X}\right)\right)+c_{1}(\mathcal{E}) A .
\end{aligned}
$$

Here $\widetilde{\omega}_{i}$ are the elements of $H^{4}(X, \mathbb{Z})$ dual to $\omega_{i}$.

Again, the wrapping numbers with respect to the tilted geometry with $\operatorname{Re}\left(U_{i}\right)=\frac{1}{2}$ correspond to $\mathcal{E}=V_{a}$. Note that even in this case, with the overall normalization chosen, the quantities $q_{I}$ need not be integer-valued even though they are integer on $T^{2} \times T^{2} \times T^{2}$. By contrast, if we stick to the unimodular basis (4.12), we insert $\mathcal{E}=\widehat{V}_{a}$, and obviously even the corresponding $p_{i}$ can be half-integer. This generalizes the effective wrapping numbers constructed from the elementary winding numbers $\left(n_{i}, \hat{m}_{i}\right)$ for $T^{2} \times T^{2} \times T^{2}$ as described in the appendix.

The structure presented in this section is rather formal. For a concrete Calabi-Yau manifold and a specified anti-holomorphic involution, finding the nice symplectic basis used in this section is not an easy task. To really see that these two discrete choices in the complex structure moduli space are indeed possible, we will now discuss one non-trivial example in some more detail.

\subsection{Example: the quintic}

While in appendix A we will provide some details on the straightforward example of a toroidal orientifold, here we would like to discuss for the simplest genuine Calabi-Yau, i.e. the Quintic, how the framework summarized in the last section actually applies.

We consider the type I string compactified on the quintic, i.e. $X=\mathbb{P}_{4}[5]$, which has Hodge numbers $\left(h_{21}, h_{11}\right)=(101,1)$ and whose complexified Kähler modulus we denote as $T=-\mathcal{B}+i J$. On the dual side we get a type IIA orientifold on the mirror manifold $\mathcal{W}=\mathbb{P}_{4}[5] / \mathbb{Z}_{5}^{3}$. The sole complex structure modulus $\psi$ is visible in the general form of the hypersurface constraint surviving the $\mathbb{Z}_{5}^{3}$ orbifold

$$
Z_{1}^{5}+Z_{2}^{5}+Z_{3}^{5}+Z_{4}^{5}+Z_{5}^{5}-(5 \psi) Z_{1} Z_{2} Z_{3} Z_{5} Z_{5}=0
$$

By a coordinate transformation like $z_{1} \rightarrow \alpha z_{1}$ with $\alpha=\exp (2 \pi i / 5)$ one sees that $\psi$ and $\alpha \psi$ define equivalent manifolds, so that only the cone $0 \leq \arg (\psi)<2 \pi / 5$ respectively $z=(5 \psi)^{-5}$ are good coordinates on the complex structure moduli space. The fundamental region for $\psi$ gets further reduced by dividing by more general coordinate transformations [80]. We assume that the type IIA anti-holomorphic involution acts just by complex conjugation $\mathcal{R}: Z_{i} \rightarrow \bar{Z}_{i}$, so that the two half-lines $\arg (\psi)=0, \pi / 5$ are the two real onedimensional components of the complex structure moduli space of the orientifold model. 
In order to see how this is related to the discrete choices of the $B$-field in the mirror dual type I description, we need to know the mirror map. Luckily, for the quintic this map is explicitly known and we just need to copy and interpret the results [80, 81].

In the region $|\psi|>1, T$ is mapped to a quotient of periods

$$
U=\frac{\Phi_{1}}{\Phi_{0}}
$$

with the periods solving the Picard-Fuchs equation given by

$$
\Phi_{0}=\sum_{n=0}^{\infty} \frac{(5 n) !}{n !^{5}} \frac{1}{(5 \psi)^{5 n}}, \quad \Phi_{k}=-\frac{5}{(2 \pi i)^{k}}[\log (5 \psi)]^{k} \Phi_{0}+\tilde{\Phi}_{k}(\psi) \quad \text { for } k=1,2,3,
$$

where, like $\Phi_{0}, \tilde{\Phi}_{k}(\psi)$ is an infinite series in the variable $\psi^{-5}$. The complex structure modulus $U$ can eventually be expressed in terms of $\psi$ as

$$
U=-\frac{5}{2 \pi i}\left[\log (5 \psi)-\frac{1}{\Phi_{0}} \sum_{m=0}^{\infty} \frac{(5 m) !}{(m !)^{5}(5 \psi)^{5 m}}(\Psi(1+5 m)-\Psi(1+m))\right],
$$

where $\Psi(x)$ denotes the digamma function. Now it is clear that $\psi \simeq \psi e^{2 \pi i N / 5}$ is mapped to the periodicity $U \simeq U-N$ and that $\mathcal{R}: U \rightarrow-\bar{U}$. In addition, the half line $\arg (\psi)=0$ is mapped to $T=U=i J$ with $J \geq J_{0} \simeq 1.21$. The other half-line $\arg (\psi)=\pi / 5$ is mapped to $T=U=-1 / 2+i J$. Note that $\psi=1$ resp. $U=i J_{0}$ is a singular point in the complex structure moduli space, where the Calabi-Yau manifold develops a conifold singularity. To describe the other side of the singular point, i.e. in the region $|\psi|<1$, one is analytically continuing the periods to this region. Note that in the mirror dual type I model this region corresponds to the Landau-Ginzburg phase of the linear sigma model. In the region around the Gepner point $\psi=0$ the mirror map has the following expansion

$$
U=-\frac{1}{2}+\frac{i}{2}\left[\cot \left(\frac{\pi}{5}\right)+\frac{\Gamma^{4}\left(\frac{4}{5}\right) \Gamma\left(\frac{2}{5}\right)}{\Gamma\left(\frac{1}{5}\right) \Gamma^{4}\left(\frac{3}{5}\right)}\left(\cot \left(\frac{\pi}{5}\right)-\cot \left(\frac{2 \pi}{5}\right)\right) e^{\frac{\pi i}{5}} \psi+O\left(\psi^{2}\right)\right] .
$$

Suppressing a discussion of branch cuts and of the fundamental region of $\psi$, which can be found in the literature [80], we realize that the Gepner point $\psi=0$ corresponds to $T=U=-\frac{1}{2}+i \cot \frac{\pi}{5}$. Therefore, the Gepner point lies on the $\mathcal{B}=1 / 2$ branch, i.e. in the Type I model it is on the same branch in Kähler moduli space as the orientifolds without vector structure. The structure of the moduli space is shown in figure 3 (essentially taken from [85], see also 86]).

For the model discussed here, i.e. the Type I string on the quintic resp. the Type IIA orientifold on the mirror quintic, the Gepner model orientifold was first discussed in 87] and featured a maximally rank tadpole canceling solution with gauge group $\mathrm{SO}(20) \times \mathrm{SO}(12)$.

\section{Outlook}

In this paper we have reconsidered Type I compactifications without vector structure. We have offered several equivalent descriptions that clarify some longstanding puzzles. In particular we have shown the consistency of a 3 generation non-supersymmetric but 

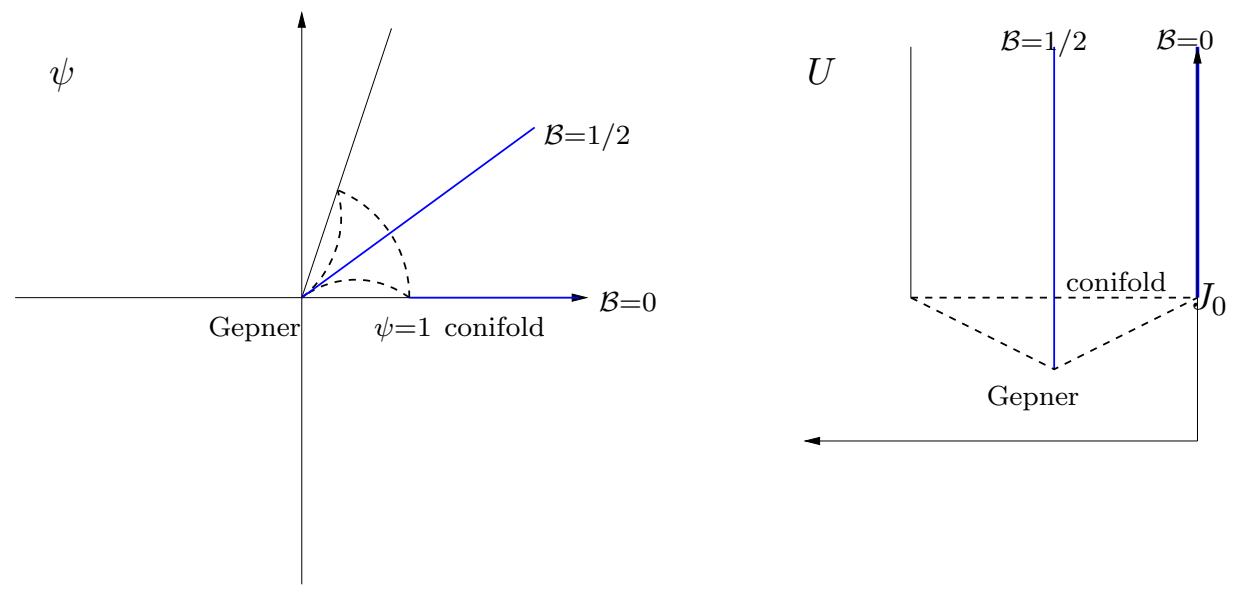

Figure 3: Complex structure moduli space for the mirror quintic $\mathcal{W} 5$ in the $\psi$-and the $U$ plane. The blue lines indicate the two discrete branches after the orientifold projection, related to $\mathcal{B}=0,1 / 2$ in the mirror dual Type I model.

tachyon-free GUT model proposed by one of us (C.B.) [19] long time ago. The possibility of relating "half-integer" wrapping numbers in the Type IIA orientifold description to a quantized NS-NS B-field opens new possibilities for model building also on non-factorisable backgrounds. As a direct application it would be interesting to apply the lessons of section 4 to type I compactifications with non-abelian bundles on genuine Calabi-Yau manifolds as considered in [75, 76]. Our results also suggest a re-analysis of toroidal compactifications with oblique fluxes [88-91] in the perspective of stabilizing off-diagonal moduli. Their mirror Type IIA description would require "co-isotropic" D-branes, i.e. wrapped rotated D-branes supporting non trivial magnetic fields associated to bundles with(out) vector structure [92, 93].

We have not explicitly considered models with different kinds of oppositely charged but mutually supersymmetric orientifold planes [94-97] that lead to models without D-branes dual to Type II models with massive R-R sector [99, 98]. Though an interesting playground in string dualities [73], at first sight this kind of models are less appealing because of the very low rank of the gauge group and the related difficulty in accomodating chiral fermions. Although model $\mathrm{C}$ is non-supersymmetric, yet it can be made non tachyonic by displacing the mutually non supersymmetric stacks along the directions where they are parallel. Moreover, one can still envisage the possibility of introducing stacks of magnetized branes mutually supersymmetric in pairs but not sharing any common global susy as a whole, see e.g. [55, 57, 59, 100].

The presence of non globally supersymmetric magnetic fields mimics the presence of lower dimensional D-branes with opposite R-R charges and may greatly help relaxing the stringent tadpole conditions on the rank of the Chan-Paton group ${ }^{12}$ and allow for further interesting lines of investigation.

\footnotetext{
${ }^{12}$ As a 'caricature' consider an (alas tachyonic) Type I model in $D=10$ with $N+16$ D9-branes and $N$ $\overline{\mathrm{D} 9}$-branes with chiral fermions and gauge symmetry 'enhancement'.
} 


\section{Acknowledgments}

We thank C. Angelantonj, M. Axenidis, V. Braun, T. Brelidze, M. Cvetič, J. Evslin, E. Floratos, A. Klemm, C. Kokorelis, R. Minasian, R. Richter, A. Sagnotti and C. Timirgaziu for useful conversations. This work has been supported in part by the European Community Human Potential Program under contracts MRTN-CT-2004-005104 and MRTN-CT-2004-512194, by the INTAS grant 03-516346, by MIUR-COFIN 2003-023852, by NATO PST.CLG.978785, by DOE grant EY-76-02-3071 and by the Excellence Cluster "The Origin and the Structure of the Universe" in Munich. C.B. thanks the ArnoldSommerfeld-Center in Munich, M.B. thanks the Ecole Normale Supérieure, R.B. thanks the University of Bonn, D.L. thanks the University of Pennsylvania and T.W. thanks the University of Wisconsin, Madison, for hospitality during part of this work.

\section{A. Toroidal example}

In this appendix we demonstrate the observations of section 4.2 for the simple example of compactifications on $T^{2} \times T^{2} \times T^{2}$. Here we have 8 homology 3 -cycles

$$
\begin{array}{ll}
A_{0}=(0,1) \otimes(0,1) \otimes(0,1), & B_{0}=(-1,0) \otimes(-1,0) \otimes(-1,0), \\
A_{1}=(-1,0) \otimes(0,-1) \otimes(0,-1), & B_{1}=(0,-1) \otimes(-1,0) \otimes(-1,0), \\
A_{2}=(0,-1) \otimes(-1,0) \otimes(0,-1), & B_{2}=(-1,0) \otimes(0,-1) \otimes(-1,0), \\
A_{3}=(0,-1) \otimes(0,-1) \otimes(-1,0), & B_{3}=(-1,0) \otimes(-1,0) \otimes(0,-1) .
\end{array}
$$

They satisfy $A_{I} \cap B_{J}=\delta_{I J}$. We also introduce the dual basis $\left(\alpha_{I}, \beta_{J}\right)$ with $\int \alpha_{I} \wedge \beta_{J}=\delta_{I J}$

$$
\begin{array}{ll}
\alpha_{0}=d y^{1} \wedge d y^{2} \wedge d y^{3}, & \beta_{0}=d x^{1} \wedge d x^{2} \wedge d x^{3}, \\
\alpha_{1}=-d x^{1} \wedge d y^{2} \wedge d y^{3}, & \beta_{1}=d y^{1} \wedge d x^{2} \wedge d x^{3}, \\
\alpha_{2}=-d y^{1} \wedge d x^{2} \wedge d y^{3}, & \beta_{2}=d x^{1} \wedge d y^{2} \wedge d x^{3}, \\
\alpha_{3}=-d y^{1} \wedge d y^{2} \wedge d x^{3}, & \beta_{3}=d x^{1} \wedge d x^{2} \wedge d y^{3} .
\end{array}
$$

The orientifold plane is chosen along the $x$-direction in each $T^{2}$ so that indeed $A_{0}, B_{i} \in$ $H_{3}^{-}\left(T^{6}, \mathbb{Z}\right)$ and $B_{0}, A_{i} \in H_{3}^{+}\left(T^{6}, \mathbb{Z}\right)$. The holomorphic coordinates

$$
d z^{i}=-U_{i} d x^{i}+d y^{i}, \quad d \bar{z}^{i}=-\bar{U}_{i} d x^{i}+d y^{i}
$$

are determined by the complex structure moduli $U^{i}$. We take $U_{i}=\left(-b_{i}+i u_{i}\right)$ with $u_{i}=\frac{R_{x}^{i}}{R_{y}^{i}}$ in terms of the radii of the elementary 1-cycles.

In the symplectic basis (A.2), the holomorphic three-form $\Omega_{3}=d z^{1} \wedge d z^{2} \wedge d z^{3}$ enjoys the expansion

$$
\Omega_{3}=\alpha_{0}+\sum_{i=1}^{3}\left(U_{i}\right) \alpha_{i}+\frac{1}{2} \sum_{i \neq k \neq j}\left(U_{i} U_{j}\right) \beta_{k}-U_{1} U_{2} U_{3} \beta_{0} .
$$


Note that indeed the ratio of periods $\frac{\int_{A_{i}} \Omega_{3}}{\int_{A_{0}} \Omega_{3}}=U_{i}$. The orientifold rule $\Omega \mathcal{R}: U_{i} \rightarrow \bar{U}_{i}$ together with the identification $U_{i} \simeq U_{i}-1$ translate into

$$
U_{i}=-\bar{U}_{i}-n
$$

Indeed, the values $U_{i}=i$ and $U_{i}=i-\frac{1}{2}$ of the untilted and tilted case satisfy this with $n=0$ and $n=1$, respectively.

One way to describe consistent 3-cycles on the torus is by introducing effective wrapping numbers $q_{I}, p_{I}$ as in equ. (4.17) with respect to the untilted basis A.1, which, for $b_{i}=1 / 2$, takes values only in $H^{2}\left(T^{6}, \mathbb{Q}\right)$. For factorizable branes these are given in terms of the wrapping numbers along the horizontal and vertical axes, $n_{i}$ and $\tilde{m}_{i}=m_{i}+b_{i} n_{i}$, by

$$
\begin{aligned}
& p_{0}=n^{1} n^{2} n^{3}, \quad p_{1}=\hat{m}^{1} n^{2} n^{3}, \quad p_{2}=n^{1} \hat{m}^{2} n^{3}, \quad p_{3}=n^{1} n^{2} \hat{m}^{3}, \\
& q_{0}=\hat{m}^{1} \hat{m}^{2} \hat{m}^{3}, \quad q_{1}=-n^{1} \hat{m}^{2} \hat{m}^{3}, \quad q_{2}=-\hat{m}^{1} n^{2} \hat{m}^{3}, \quad q_{3}=-\hat{m}^{1} \hat{m}^{2} n^{3} .
\end{aligned}
$$

This is in agreement with the general expression (4.25).

\section{References}

[1] M. Berkooz et al., Anomalies, dualities and topology of $D=6 N=1$ superstring vacua, Nucl. Phys. B 475 (1996) 115 hep-th/9605184.

[2] M. Bianchi, A note on toroidal compactifications of the type-I superstring and other superstring vacuum configurations with 16 supercharges, Nucl. Phys. B 528 (1998) 73 hep-th/9711201.

[3] E. Witten, Toroidal compactification without vector structure, JHEP 02 (1998) 006 hep-th/9712028.

[4] P.S. Aspinwall, Point-like instantons and the $\operatorname{Spin}(32) / Z_{2}$ heterotic string, Nucl. Phys. B 496 (1997) 149 hep-th/9612108.

[5] A. Sen and S. Sethi, The mirror transform of type-I vacua in six dimensions, Nucl. Phys. B 499 (1997) 45 hep-th/9703157.

[6] W. Lerche, C. Schweigert, R. Minasian and S. Theisen, A note on the geometry of CHL heterotic strings, Phys. Lett. B 424 (1998) 53 hep-th/9711104].

[7] Z. Kakushadze, G. Shiu and S.H.H. Tye, Type IIB orientifolds with NS-NS antisymmetric tensor backgrounds, Phys. Rev. D 58 (1998) 086001 hep-th/9803141.

[8] C. Angelantonj and R. Blumenhagen, Discrete deformations in type-I vacua, Phys. Lett. B $473(2000) 86$ hep-th/9911190.

[9] C. Angelantonj, Comments on open-string orbifolds with a non-vanishing $B_{a b}$, Nucl. Phys. B $\mathbf{5 6 6}(2000) 126$ hep-th/9908064.

[10] C. Angelantonj, R. Blumenhagen and M.R. Gaberdiel, Asymmetric orientifolds, brane supersymmetry breaking and non-BPS branes, Nucl. Phys. B 589 (2000) 545 hep-th/0006033.

[11] Z. Kakushadze, Geometry of orientifolds with NS-NS B-flux, Int. J. Mod. Phys. A 15 (2000) 3113 hep-th/0001212]. 
[12] A. Keurentjes, Orientifolds and twisted boundary conditions, Nucl. Phys. B 589 (2000) 440 hep-th/0004073.

[13] J. de Boer et al., Triples, fluxes and strings, Adv. Theor. Math. Phys. 4 (2002) 995 hep-th/0103170.

[14] A. Keurentjes, Discrete moduli for type-I compactifications, Phys. Rev. D 65 (2002) 026007 hep-th/0105101.

[15] M. Bianchi and A. Sagnotti, Open strings and the relative modular group, Phys. Lett. B 231 (1989) 389 .

[16] M. Bianchi and A. Sagnotti, On the systematics of open string theories, Phys. Lett. B 247 (1990) 517 .

[17] M. Bianchi and A. Sagnotti, Twist symmetry and open string Wilson lines, Nucl. Phys. B 361 (1991) 519.

[18] M. Bianchi, G. Pradisi and A. Sagnotti, Toroidal compactification and symmetry breaking in open string theories, Nucl. Phys. B 376 (1992) 365.

[19] C. Bachas, A way to break supersymmetry, hep-th/9503030.

[20] R. Blumenhagen, L. Görlich, B. Körs and D. Lüst, Noncommutative compactifications of type-I strings on tori with magnetic background flux, JHEP 10 (2000) 006 hep-th/0007024.

[21] C. Angelantonj, I. Antoniadis, E. Dudas and A. Sagnotti, Type-I strings on magnetised orbifolds and brane transmutation, Phys. Lett. B 489 (2000) 223 hep-th/0007090.

[22] R. Blumenhagen, B. Körs and D. Lüst, Type I strings with F- and B-flux, JHEP 02 (2001) 030 hep-th/0012156.

[23] C. Angelantonj and A. Sagnotti, Open strings, Phys. Rept. 371 (2002) 1] [Erratum ibid. 376 (2003) 339] hep-th/0204089.

[24] A.M. Uranga, Chiral four-dimensional string compactifications with intersecting D-branes, Class. and Quant. Grav. 20 (2003) S373 hep-th/0301032.

[25] E. Kiritsis, D-branes in standard model building, gravity and cosmology, Fortschr. Phys. 52 (2004) 200 Phys. Rept. 421 (2005) 105 [Erratum ibid. 429 (2006) 121] hep-th/0310001.

[26] R. Blumenhagen, M. Cvetič, P. Langacker and G. Shiu, Toward realistic intersecting D-brane models, Ann. Rev. Nucl. Part. Sci. 55 (2005) 71 hep-th/0502005.

[27] R. Blumenhagen, B. Körs, D. Lüst and S. Stieberger, Four-dimensional string compactifications with D-branes, orientifolds and fluxes, Phys. Rept. 445 (2007) 1 hep-th/0610327.

[28] I. Pesando, A comment on discrete Kalb-Ramond field on orientifold and rank reduction, arXiv:0804.3931.

[29] O.J. Ganor, S. Ramgoolam and W. Taylor, Branes, fluxes and duality in M(atrix)-theory, Nucl. Phys. B 492 (1997) 191 hep-th/9611202.

[30] R. Rabadán, Branes at angles, torons, stability and supersymmetry, Nucl. Phys. B 620 (2002) 152 hep-th/0107036.

[31] G. 't Hooft, A property of electric and magnetic flux in nonabelian gauge theories, Nucl. Phys. B 153 (1979) 141. 
[32] R. Friedman, J. Morgan and E. Witten, Vector bundles and F-theory, Commun. Math. Phys. 187 (1997) 679 hep-th/9701162.

[33] M. Bianchi and Y.S. Stanev, Open strings on the Neveu-Schwarz pentabrane, Nucl. Phys. B 523 (1998) 193 hep-th/9711069.

[34] C. Bachas, M.R. Douglas and C. Schweigert, Flux stabilization of D-branes, JHEP 05 (2000) 048 hep-th/0003037.

[35] W. Taylor, D2-branes in B fields, JHEP 07 (2000) 039 hep-th/0004141.

[36] A. Alekseev, A. Mironov and A. Morozov, On B-independence of RR charges, Phys. Lett. B $532(2002) 350$ hep-th/0005244.

[37] D. Marolf, Chern-Simons terms and the three notions of charge, hep-th/0006117.

[38] J.M. Figueroa-O'Farrill and S. Stanciu, D-brane charge, flux quantization and relative (co)homology, JHEP 01 (2001) 006 hep-th/0008038.

[39] E. Witten, Dyons of charge e $\theta / 2 \pi$, Phys. Lett. B 86 (1979) 283.

[40] S. Chaudhuri, G. Hockney and J.D. Lykken, Maximally supersymmetric string theories in $D<10$, Phys. Rev. Lett. 75 (1995) 2264 hep-th/9505054.

[41] I. Antoniadis, C.P. Bachas and C. Kounnas, Four-dimensional superstrings, Nucl. Phys. B 289 (1987) 87.

[42] A. Collinucci, F. Denef and M. Esole, D-brane deconstructions in IIB orientifolds, arXiv:0805.1573.

[43] R. Blumenhagen, J.P. Conlon and K. Suruliz, Type IIA orientifolds on general supersymmetric $\mathbb{Z}_{N}$ orbifolds, JHEP 07 (2004) 022 hep-th/0404254.

[44] S. Förste, C. Timirgaziu and I. Zavala, Orientifold's landscape: non-factorisable six-tori, JHEP 10 (2007) 025 arXiv:0707.0747.

[45] J. Polchinski and E. Witten, Evidence for heterotic - type I string duality, Nucl. Phys. B 460 (1996) 525 hep-th/9510169.

[46] A. Sen, $\mathrm{SO}(32)$ spinors of type-I and other solitons on brane-antibrane pair, JHEP 09 (1998) 023 hep-th/9808141.

[47] E. Witten, D-branes and k-theory, JHEP 12 (1998) 019 hep-th/9810188.

[48] R. Minasian and G.W. Moore, K-theory and Ramond-Ramond charge, JHEP 11 (1997) 002 hep-th/9710230.

[49] E. Witten, An SU(2) anomaly, Phys. Lett. B 117 (1982) 324.

[50] A.M. Uranga, D-brane probes, RR tadpole cancellation and k-theory charge, Nucl. Phys. B 598 (2001) 225 hep-th/0011048.

[51] N. Arkani-Hamed and S. Dimopoulos, Supersymmetric unification without low energy supersymmetry and signatures for fine-tuning at the LHC, JHEP 06 (2005) 073 hep-th/0405159.

[52] G.F. Giudice and A. Romanino, Split supersymmetry, Nucl. Phys. B 699 (2004) 65 [Erratum ibid. B 706 (2005) 65] hep-ph/0406088. 
[53] I. Antoniadis, K. Benakli, A. Delgado, M. Quirós and M. Tuckmantel, Split extended supersymmetry from intersecting branes, Nucl. Phys. B 744 (2006) 156 hep-th/0601003.

[54] J.R. Ellis, P. Kanti and D.V. Nanopoulos, Intersecting branes flip SU(5), Nucl. Phys. B 647 (2002) 235 hep-th/0206087.

[55] C. Kokorelis, Deformed intersecting D6-brane GUTs. I, JHEP 11 (2002) 027 hep-th/0209202.

[56] M. Cvetič, I. Papadimitriou and G. Shiu, Supersymmetric three family SU(5) grand unified models from type IIA orientifolds with intersecting D6-branes, Nucl. Phys. B 659 (2003) 193 [Erratum ibid. B 696 (2004) 298] hep-th/0212177].

[57] M. Axenides, E. Floratos and C. Kokorelis, SU(5) unified theories from intersecting branes, JHEP 10 (2003) 006 hep-th/0307255.

[58] G.K. Leontaris and J. Rizos, A D-brane inspired $\mathrm{U}(3)_{C} \times \mathrm{U}(3)_{L} \times \mathrm{U}(3)_{R}$ model, Phys. Lett. B 632 (2006) 710 hep-ph/0510230.

[59] E. Floratos and C. Kokorelis, MSSM GUT string vacua, split supersymmetry and fluxes, hep-th/0607217.

[60] E. Witten, Small instantons in string theory, Nucl. Phys. B 460 (1996) 541 hep-th/9511030.

[61] E.G. Gimon and J. Polchinski, Consistency conditions for orientifolds and D-manifolds, Phys. Rev. D 54 (1996) 1667 hep-th/9601038.

[62] E. Witten, World-sheet corrections via D-instantons, JHEP 02 (2000) 030 hep-th/9907041.

[63] R. Blumenhagen, M. Cvetič and T. Weigand, Spacetime instanton corrections in $4 D$ string vacua - the seesaw mechanism for D-brane models, Nucl. Phys. B 771 (2007) 113 hep-th/0609191.

[64] L.E. Ibáñez and A.M. Uranga, Neutrino Majorana masses from string theory instanton effects, JHEP 03 (2007) 052 hep-th/0609213.

[65] M. Bianchi and E. Kiritsis, Non-perturbative and flux superpotentials for type I strings on the $Z_{3}$ orbifold, Nucl. Phys. B 782 (2007) 26 hep-th/0702015.

[66] M. Cvetič and T. Weigand, Hierarchies from D-brane instantons in globally defined Calabi-Yau orientifolds, arXiv:0711.0209.

[67] C. Beasley and E. Witten, New instanton effects in string theory, JHEP 02 (2006) 060 hep-th/0512039.

[68] R. Blumenhagen, M. Cvetič, R. Richter and T. Weigand, Lifting D-instanton zero modes by recombination and background fluxes, JHEP 10 (2007) 098 arXiv:0708.0403.

[69] C. Bachas, C. Fabre, E. Kiritsis, N.A. Obers and P. Vanhove, Heterotic/type-I duality and D-brane instantons, Nucl. Phys. B 509 (1998) 33 hep-th/9707126.

[70] E. Kiritsis and N.A. Obers, Heterotic/type-I duality in $D<10$ dimensions, threshold corrections and D-instantons, JHEP 10 (1997) 004 hep-th/9709058].

[71] C. Bachas, Heterotic versus type-I, Nucl. Phys. 68 (Proc. Suppl.) (1998) 348 hep-th/9710102.

[72] W. Lerche, Elliptic index and superstring effective actions, Nucl. Phys. B 308 (1988) 102. 
[73] M. Bianchi, E. Gava, F. Morales and K.S. Narain, D-strings in unconventional type-I vacuum configurations, Nucl. Phys. B 547 (1999) 96 hep-th/9811013.

[74] M. Bianchi and J.F. Morales, Unoriented D-brane instantons vs heterotic worldsheet instantons, JHEP 02 (2008) 073 arXiv:0712.1895.

[75] R. Blumenhagen, G. Honecker and T. Weigand, Supersymmetric (non-)abelian bundles in the type-I and $\mathrm{SO}(32)$ heterotic string, JHEP 08 (2005) 009 hep-th/0507041.

[76] R. Blumenhagen, G. Honecker and T. Weigand, Non-abelian brane worlds: the heterotic string story, JHEP 10 (2005) 086 hep-th/0510049.

[77] R. Blumenhagen, V. Braun, B. Körs and D. Lüst, Orientifolds of K3 and Calabi-Yau manifolds with intersecting D-branes, JHEP 07 (2002) 026 hep-th/0206038.

[78] T.W. Grimm and J. Louis, The effective action of type IIA Calabi-Yau orientifolds, Nucl. Phys. B 718 (2005) 153 hep-th/0412277.

[79] R. Blumenhagen, F. Gmeiner, G. Honecker, D. Lüst and T. Weigand, The statistics of supersymmetric D-brane models, Nucl. Phys. B 713 (2005) 83 hep-th/0411173.

[80] P. Candelas, X.C. De La Ossa, P.S. Green and L. Parkes, A pair of Calabi-Yau manifolds as an exactly soluble superconformal theory, Nucl. Phys. B 359 (1991) 21.

[81] P. Candelas, X.C. De la Ossa, P.S. Green and L. Parkes, An exactly soluble superconformal theory from a mirror pair of Calabi-Yau manifolds, Phys. Lett. B 258 (1991) 118.

[82] P.S. Aspinwall, D-branes on Calabi-Yau manifolds, hep-th/0403166.

[83] S. Hosono, A. Klemm and S. Theisen, Lectures on mirror symmetry, hep-th/9403096.

[84] M.R. Douglas, R. Reinbacher and S.T. Yau, Branes, bundles and attractors: Bogomolov and beyond, math.AG/0604597.

[85] P.S. Aspinwall, The moduli space of $N=2$ superconformal field theories, hep-th/9412115.

[86] I. Brunner, K. Hori, K. Hosomichi and J. Walcher, Orientifolds of Gepner models, JHEP 02 (2007) 001 hep-th/0401137.

[87] R. Blumenhagen and A. Wisskirchen, Spectra of $4 D, N=1$ type-I string vacua on non-toroidal CY threefolds, Phys. Lett. B 438 (1998) 52 hep-th/9806131.

[88] I. Antoniadis and T. Maillard, Moduli stabilization from magnetic fluxes in type-I string theory, Nucl. Phys. B 716 (2005) 3 hep-th/0412008.

[89] M. Bianchi and E. Trevigne, The open story of the magnetic fluxes, JHEP 08 (2005) 034 hep-th/0502147.

[90] I. Antoniadis, A. Kumar and T. Maillard, Moduli stabilization with open and closed string fluxes, hep-th/0505260.

[91] M. Bianchi and E. Trevigne, Gauge thresholds in the presence of oblique magnetic fluxes, JHEP 01 (2006) 092 hep-th/0506080.

[92] A. Font, L.E. Ibáñez and F. Marchesano, Coisotropic D8-branes and model-building, JHEP 09 (2006) 080 hep-th/0607219.

[93] P. Anastasopoulos, M. Bianchi, G. Sarkissian and Y.S. Stanev, On gauge couplings and thresholds in type-I Gepner models and otherwise, JHEP 03 (2007) 059 hep-th/0612234. 
[94] A. Hanany and B. Kol, On orientifolds, discrete torsion, branes and M-theory, JHEP 06 (2000) 013 hep-th/0003025.

[95] S. Sugimoto, Anomaly cancellations in type-I D9-Dי system and the USp(32) string theory, Prog. Theor. Phys. 102 (1999) 685 hep-th/9905159.

[96] O. Bergman, E.G. Gimon and S. Sugimoto, Orientifolds, $R R$ torsion and k-theory, JHEP 05 (2001) 047 hep-th/0103183.

[97] E. Dudas, J. Mourad and A. Sagnotti, Charged and uncharged D-branes in various string theories, Nucl. Phys. B 620 (2002) 109 hep-th/0107081.

[98] C. Angelantonj, M. Bianchi, G. Pradisi, A. Sagnotti and Y.S. Stanev, Comments on Gepner models and type-I vacua in string theory, Phys. Lett. B 387 (1996) 743 hep-th/9607229].

[99] C. Vafa and E. Witten, Dual string pairs with $N=1$ and $N=2$ supersymmetry in four dimensions, Nucl. Phys. 46 (Proc. Suppl.) (1996) 225 hep-th/9507050.

[100] R. Emparan and G.T. Horowitz, Microstates of a neutral black hole in M-theory, Phys. Rev. Lett. 97 (2006) 141601 hep-th/0607023. 